\title{
Regularization Theory in Image Restoration-The Stabilizing Functional Approach
}

\author{
NICOLAOS B. KARAYIANNIS, STUDENT MEMBER, IEEE, AND \\ ANASTASIOS N. VENETSANOPOULOS, FELLOW, IEEE
}

\begin{abstract}
This paper presents several aspects of the application of regularization theory in image restoration. This is accomplished by extending the applicability of the stabilizing functional approach to 2-D ill-posed inverse problems. Image restoration is formulated as the constrained minimization of a stabilizing functional. The choice of a particular quadratic functional to be minimized is related to the a priori knowledge regarding the original object through a formulation of image restoration as a maximum a posteriori estimation problem. This formulation is based on image representation by certain stochastic partial differential equation image models. The analytical study and computational treatment of the resulting optimization problem are subsequently presented. As a result, a variety of regularizing filters and iterative regularizing algorithms are proposed. A relationship between the regularized solutions proposed and optimal Wiener estimation is also identified. The filters and algorithms proposed are evaluated through several experimental results.
\end{abstract}

\section{INTRODUCTION}

$\mathrm{T}_{\mathrm{b}}$ HE image formation process is commonly formulated by the following linear model [1], [2]:

$$
\text { Af }+n=g
$$

where $f$ is the original object, $g$ is the observed image, and $n$ is the additive noise due to the image recording system. $A$ is a linear operator describing the degradation associated with the image formation. Image restoration is the process of inferring the best possible estimate for the original object $f$, given the observed image $g$. When the image formation is described by a continuous spacevariant linear model, (1.1) corresponds to the following integral equation [1]:

$$
\iint h(u, v, x, y) f(x, y) d x d y+n(u, v)=g(u, v)
$$

where $h(\cdot)$ is a function of four variables, describing positions in both object and image spaces, also known as point spread function (PSF) in the literature [1]. In the case of a space-invariant continuous formulation, (1.2) corresponds to 2-D convolution. When the image formation is described by a discrete model, (1.1) corresponds to the following matrix-vector equation [1]:

$$
A f+n=g \text {. }
$$

Manuscript received June 1, 1988; revised August 2, 1989.

The authors are with the Department of Electrical Engineering, University of Toronto, Toronto, ON, Canada M5S 1A4.

IEEE Log Number 9035657
In (1.3), $f$ and $g$ are vectors consisting of the lexicographically ordered elements of the object and image, respectively, and $\boldsymbol{n}$ is the noise vector. The degradation associated with the image formation is described in (1.3) by the matrix $\boldsymbol{A}$, also referred to as point spread matrix $(P S M)$ in the literature [1].

An inverse problem is characterized as ill-posed when there is no guarantee for the existence, uniqueness, and stability of the solution based on direct inversion. The solution of an inverse problem is not guaranteed to be stable if a small perturbation of the data can produce a large effect in the solution. Image restoration belongs to a general class of problems that were rigorously classified as ill-posed problems in [3]. An even more intuitive reasoning about the ill-posed nature of the problem can be found in [4]. Regularization theory (RT) provides a formal basis for the development of regularized solutions of ill-posed problems. The basic elements of RT were presented by Tikhonov et al. in [3]. In [5], Nashed emphasized the relationship between regularization and generalized inverses. The stabilizing functional approach is one of the basic methodologies for the development of regularized solutions [3]. According to this approach, an ill-posed problem can be formulated as the constrained minimization of a certain functional, called stabilizing functional. The specific constraints imposed by the stabilizing functional approach on the solution depend on the form and the properties of the stabilizing functional used. From the nature of the problem, these constraints are necessarily related to the a priori information regarding the expected regularized solution.

Although the basic concepts within RT are well defined, there exists some confusion in the literature regarding RT. The use of the word "regularization" by some authors generates often the false impression that RT is restricted to the minimum norm solution of ill-posed inverse problems only. On the other hand, it seems to be commonly believed that RT is restricted to the stabilizing functional approach only. However, a variety of alternative regularized solutions can be determined on the basis of the general and even more abstract concept of the regularizing operator ( $R O)$ [ [3]. In fact, the stabilizing functional approach is a particular methodology in constructing RO's. An extensive study of the application of the RO approach in image restoration was presented by the authors in [6]. 
One of the earliest image restoration approaches motivated by $\mathrm{RT}$ was the application of cubic splines in image restoration, presented by Hou and Andrews in [7]. Some remarks regarding the application of RT in image restoration were also reported by Hunt in [4]. More recently, Katsaggelos et al. presented an image restoration approach using some elements of RT [8]. However, the number of image restoration approaches that were directly motivated by RT is only a small portion of the image restoration approaches that can be related to RT. In fact, there exist several constrained image restoration approaches that can be classified as special applications of the stabilizing functional approach. The simplest, and rather primitive, one is the minimum norm image restoration approach. In this case, the stabilizing functional is simply the norm of the original object. It is shown in this paper that the entire family of constrained least squares approaches [9] can be related to the stabilizing functional approach. More specifically, any constrained least squares formulation of the image restoration problem can be obtained from the stabilizing functional approach by using a quadratic stabilizing functional of a special form. Finally, the relationship between maximum entropy (ME) image restoration [10], [11] and the stabilizing functional approach is of particular interest. The stabilizing functional approach amounts to the ME formulation of the image restoration problem, provided that the negative entropy functional is used as stabilizing functional. The above relationship is a clear indication that, in general, the stabilizing functional approach amounts to nonlinear regularized solutions of image restoration. The computational requirements associated with the implementation of ME image restoration, in conjunction with the huge amount of data typically involving in image restoration, provide an indication of the computational difficulties that could arise from the use of global nonquadratic stabilizing functionals.

Image restoration is not the only inverse problem that can be formulated as the minimization of a certain functional, or equivalently, as variational principles. Variational principles recently attracted the attention of many researchers in the area of image processing and low level computer vision [12]. Some of the problems formulated using variational principles were edge detection [13]-[15], determination of optical flow [16], and the shape from shading problem [17]. The use of variational principles in visual surface interpolation, originally proposed by Grimson in [18] and [19], is of particular interest here. In [19], the visual surface interpolation problem was formulated as the constrained minimization of a certain quadratic functional. A computational treatment of the resulting optimization problem was reported by Grimson in [20]. The role of quadratic functionals based on rotationally symmetric differential operators was studied by Brady and Horn in [21]. In [22] and [23], Terzopoulos presented the study of several analytical and computational aspects regarding the application of variational principles in visual surface interpolation.

The minimization of a quadratic functional is the common point of all the above approaches. In [24], Terzo- poulos presented some arguments regarding the undesirable effects caused by the use of quadratic functionals in visual reconstruction problems that arise in early vision. One of the basic ones was that the global smoothness constraints imposed by the use of quadratic functionals cannot guarantee the preservation of certain discontinuities that play an important role in vision. Based on this argument, he proposed a class of stabilizing functionals for piecewise continuous regularization, called controlledcontinuity stabilizers. In addition to a general analytical study regarding the use of such stabilizers, he also discussed several alternatives related to the computational treatment of the resulting optimization problem. Although the original idea is an attractive alternative to standard regularization, it seems that there exist certain difficulties in the application level. In other words, the derivation of clearly specified and easily reproducible algorithms based on such stabilizers is still an open problem. In particular, a possible use of such stabilizers in image restoration should necessarily overcome certain problems related to the local nature of the constraints imposed by such a formulation, given the representation of the image formation by a global model.

In a recent publication [25], Pavlidis reported some useful comments on the use of variational principles in image processing and low level computer vision. According to this author, one of the most important problems to be investigated is the justification of the choice of a particular functional in a given application. The necessary constraint in choosing the functional to be minimized is the one imposed by the mathematical problem itself, that is, the requirement that the functional to be chosen possesses a minimum. Another important factor that usually affects the choice of a particular functional relates to the analytical difficulties and computational requirements resulting from this particular choice. However, there are not generally accepted rules for the particular choice of a functional that reflects the nature of the given physical problem. To date, the formulation of certain problems as variational principles was mostly based on the constrained minimization of quadratic functionals. The only attempt to introduce nonquadratic functionals emphasizing local features of the image was the one, mentioned above, by Terzopoulos. On the other hand, ME image restoration can be interpreted as the only image restoration approach that is based on the minimization of a global nonquadratic functional. In general, there exist several problems associated with the use of global nonquadratic functionals. One of the most important problems is the justification of the use of a particular nonquadratic functional. In fact, there is no guarantee that the use of a global nonquadratic functional can efficiently prevent local discontinuities in the image. This seems to be one of the reasons for the lack of a generally accepted nonlinear theory in image restoration. On the other hand, the global model that describes the image formation clearly distinguishes image restoration from other similar problems as, for instance, the problem of image filtering when there is no distortion associated with the image formation but additive noise. Most of the proposed nonlinear filtering techniques were 
based on local processing by employing certain nonlinear operators that can effectively reduce the noise, while simultaneously preserve local discontinuities [26]. Such nonlinear techniques provided remarkable results, at least for some kinds of non-Gaussian noise. However, the application of local nonlinear processing in image restoration is restricted by the requirement that the resulting restoration technique should also efficiently deal with the global nature of the inversion problem. Not surprisingly, it is particularly difficult to identify in the literature any particular nonlinear image restoration technique that is widely accepted, or used as a reference by the researchers in the area.

This paper is organized as follows: In Section II, image restoration is formulated on the basis of the stabilizing functional approach as the constrained minimization of a certain stabilizing functional. The remainder of this section presents a systematic search for stabilizing functionals among the elements of a general class of functionals. The study of a certain class of quadratic functionals is particularly emphasized. Section III presents an extensive study of the unconstrained minimization problem, obtained from the original constrained one using the method of Lagrange multipliers. The minimization problem resulting from the continuous formulation of the image restoration problem is initially studied. The remainder of this section is devoted to the discrete formulation of the problem. The discrete approximation of a certain class of quadratic functionals is obtained by using the finite element method. In Section IV, the selection of specific stabilizing functionals is based on a particular formulation of image restoration as a maximum a posteriori (MAP) estimation problem. This formulation allows a stochastic interpretation of the strictly deterministic stabilizing functional approach. As a result, the choice of stabilizing functionals is related to the image representation by stochastic partial differential equation (SPDE) image models. Section V presents the derivation of a class of Regularizing Filters (RF's) based on the stabilizing functional approach. A generalization of this class of filters is subsequently proposed, resulting in a class of generalized regularizing filters (GRF's). Section VI presents the derivation of several well-determined and easily reproducible algorithms for discrete image restoration. Section VII investigates the relationship between the class of regularized solutions proposed in this paper and optimal Wiener estimation. In Section VIII, several experimental results are presented, compared, and discussed. Section IX contains concluding remarks.

\section{The Stabilizing Functional Approach}

\section{A. Stabilizing Functionals}

A continuous functional is a mapping from a space of functions to the real numbers. In particular, a nonnegative functional is a mapping to the nonnegative real numbers. It was proposed in [3] that the solution of ill-posed inverse problems can be achieved by the constrained minimization of a functional satisfying certain properties, called stabilizing functional. Consider the inverse problem defined by (1.1). The concept of the stabilizing functional is now introduced [3].

Let $\Omega[f]$ be the continuous nonnegative functional defined on a subspace $\bar{F}$ of a certain space $F$, and

a) the solution $f^{*}$ of $(1.1)$ belongs to the domain of definition of $\Omega[f]$;

b) for every positive $\epsilon$, the set of elements $f$ of $\bar{F}$ for which $\Omega[f] \leq \epsilon$ is a compact subset in $\bar{F}$.

Any functional $\Omega[f]$ defined on $\bar{F}$ and satisfying the above two properties is called stabilizing functional. According to the stabilizing functional approach, the problem of determining regularized solutions of (1.1) can be formulated as follows. Determine the $f=f^{*}$ in $\bar{F}$, which minimizes the functional $\Omega[f]$, under the condition that the element $f=f^{*}$ satisfies the equation

$$
\left\|A f^{*}-g\right\|=\delta
$$

where $\|\cdot\|$ is the $L_{2}$-norm and $\delta$ is a positive constant to be chosen.

The definition of the stabilizing functional given above is very general. However, an open question remains unanswered concerning the choice of stabilizing functionals for 2-D ill-posed problems. Since the choice of the stabilizing functional or the class of stabilizing functionals is the key issue in this approach, the problem is further investigated. In the analysis which follows, a general and very broad class of functionals is considered as the source of possible stabilizing functionals.

Let $C^{(K, L)}[R]$ be the space of all continuous smooth functions (i.e., continuous functions with continuous partial derivatives up to order $(K, L))$ in the region of interest $R$. Throughout the paper, the partial derivatives will be denoted as follows:

$$
\frac{\partial^{p+q} f}{\partial x^{p} \partial y^{q}}=f_{x^{p} y^{q}}=D_{x}^{p} D_{y}^{q} f
$$

Let $\Gamma$ be the boundary of the region of interest $R$. In addition, consider the space $C_{0}^{(K . L)}[R]$ defined as follows:

$$
\begin{aligned}
C_{0}^{(K, L)}[R] & \equiv\left\{f / f \in C^{(K . L)}[R]:\left.\frac{\partial^{p+q} f}{\partial x^{p} \partial y^{q}}\right|_{\Gamma=0},\right. \\
(p, q) & =(0,0), \cdots,(K-1, L-1)\}
\end{aligned}
$$

The space $C_{0}^{(K, L)}[R]$ consists of continuous smooth functions in $R$, whose partial derivatives vanish at the boundary $\Gamma$ of $R$. For any function $f \in C^{(K, L)}[R]$, consider the class of functionals $\Phi$ consisting of elements of the following form:

$$
\begin{aligned}
& \Omega[f] \\
& \quad=\iint F\left(x, y,\left.\frac{\partial^{p+q} f}{\partial x^{p} \partial y^{q}}\right|_{(p . q)=(0.0), \cdots,(K . L)}\right) d x d y .
\end{aligned}
$$


In $(2.4), F(\cdot)$ can be any twice continuously differentiable function with respect to $f$ and its partial derivatives up to any desired order $(K, L)$.

The necessary condition for a function $f=\hat{f}$ to an extremum for $\Omega[f]$ is that it satisfies the equation

$$
\delta \Omega[h]=0 \forall h \in C_{0}^{(K, L)}[R]
$$

where $\delta \Omega[\cdot]$ is the first variation of $\Omega[f]$ [27], [28]. Equation (2.5) results in what is known as the Euler-Lagrange equation [28]. It was shown in [29] that the first variation of any functional of the form (2.4) is given by

$\delta \Omega[h]=2\left\langle h, \Omega^{\prime}[f]\right\rangle=2 \iint h \Omega^{\prime}[f] d x d y$

where $h$ is any admissible function, i.e., $h \in C_{0}^{(K, L)}[R]$, and

$$
\Omega^{\prime}[f]=\frac{1}{2} \sum_{p=0}^{K} \sum_{q=0}^{L}(-1)^{p+q} \frac{\partial^{p+q}}{\partial x^{p} \partial y^{q}}\left(\frac{\partial F}{\partial f_{x^{p} y^{q}}}\right) .
$$

The necessary condition for an extremum to be a minimum depends on the sign of the second variation of $\Omega[f]$, denoted here as $\delta^{2} \Omega[\cdot]$. That is, the necessary condition for a functional $\Omega[f] \in \Phi$ to have a minimum for $f=\hat{f} \in C^{(K, L)}[R]$ is that, for $f=\hat{f}$

$$
\delta^{2} \Omega[h] \geq 0 \forall h \in C_{0}^{(K, L)}[R] .
$$

The condition for the existence of a minimum given by (2.8) is very general; however, it cannot be easily applied in practical situations. Therefore, for a certain class of functionals, it should be replaced by a more specific and applicable condition. This can be achieved by appropriately developing the definition of the second variation of functionals [27], [28]. A basic, although simple, example is the necessary condition for one-variable functionals of a specific form known as Legendre theorem [27]. An analogous condition, appropriate for the class of functionals defined by (2.4), was presented in [29].

The condition (2.8) is crucial for the selection of the stabilizing functionals among the elements of $\Phi$. Let $\Phi_{S}$ be the subclass of $\Phi$ defined as follows:

$$
\Phi_{S}=\left\{\Omega / \Omega[f] \in \Phi: \delta^{2} \Omega[h] \geq 0 \forall h \in C_{0}^{(K, L)}[R]\right\} .
$$

That is, $\Phi_{S}$ consists of all functionals in $\Phi$ whose second variation is nonnegative. In general, the search among the elements of $\Phi$ for the functionals belonging also to $\Phi_{S}$ is a particularly complicated problem. The interested reader can find a more detailed and elaborate treatment of this problem in [29]. For the sake of analytical simplicity, this search is restricted here to a particular type of functionals within $\Phi$, called quadratic functionals.

\section{B. Quadratic Stabilizing Functionals}

Consider the general class of quadratic functionals whose elements are of the following form:

$$
Q[f]=\iint\left(\sum_{k, m=0}^{K} \sum_{l, n=0}^{L} f_{x^{k} y^{\prime}} q_{k, l ; m, n} f_{x^{m} y^{n}}\right) d x d y
$$

where $q_{k, l ; m, n} ; 1 \leq k, m \leq K, 1 \leq l, n \leq L$ are real constant coefficients. Obviously, any functional defined by (2.10) is an element of the class $\Phi$ of functionals. Some important properties of the class of quadratic functionals defined by (2.10) are presented by the following theorem.

Theorem 1: Consider any quadratic functional of the form (2.10). For any $h \in C^{(K, L)}[R]$

$$
\delta^{2} Q[h]=Q[h] .
$$

If $q_{k, l ; m, n}=q_{m, n ; k, l}$, then for any $f \in C^{(K, L)}[R]$

$$
Q^{\prime}[f]=B\left(D_{x}, D_{y}\right) f(x, y)
$$

where $B(\cdot)$ is the following linear partial differential operator $(P D O)$

$$
B\left(D_{x}, D_{y}\right)=\sum_{k, m=0}^{K} \sum_{l, n=0}^{L}(-1)^{k+l} q_{k, l ; m, n} D_{x}^{k+m} D_{y}^{l+n} .
$$

Finally, if $f \in C_{0}^{(K, L)}[R]$, the quadratic functional of the form (2.10) can be written as follows:

$$
Q[f]=\langle f, B f\rangle
$$

where $B(\cdot)$ is the PDO defined by (2.13). $\operatorname{dix}$ A.

Proof: The proof of this theorem is given in Appen-

Among the mathematical properties of quadratic functionals, a particularly important one is the simplicity of the necessary condition for the existence of a minimum of such functionals. This condition can be derived as a special application of the general condition presented in [29]. The same condition can also be derived by using the results of the above theorem. Suppose that a certain quadratic functional of the form (2.10) is nonnegative definite, i.e.,

$$
Q[f] \geq 0 \forall f \in C^{(K, L)}[R] .
$$

Since $C_{0}^{(K, L)}[R]$ is a subspace of $C^{(K, L)}[R],(2.15)$ implies that

$$
Q[h] \geq 0 \forall h \in C_{0}^{(K, L)}[R] .
$$

Taking into account (2.11)

$$
\delta^{2} Q[h] \geq 0 \forall h \in C_{0}^{(K, L)}[R] .
$$

This is exactly the necessary condition for the existence of a minimum of a quadratic functional of the form (2.10). As a result, the extremum of any nonnegative quadratic functional is guaranteed to be a minimum. Therefore, the above theorem indicates the close relationship between the necessary condition for the existence of a minimum of a quadratic functional and its nonnegative definition. It can easily be seen that any quadratic functional of the form (2.10) is nonnegative if the matrix formed by lexico- 
graphically ordering the coefficients $q_{k, l, m, n}$ is nonnegative definite.

A very useful class of nonnegative quadratic functionals is now introduced. Suppose that

$$
q_{k, l ; m, n}=\lambda_{k, l} \lambda_{m, n} .
$$

Then, (2.10) amounts to the following class of nonnegative quadratic functionals:

$$
Q_{L}[f]=\|L f\|^{2}=\iint(L f)^{2} d x d y
$$

where

$$
\begin{aligned}
L\left(D_{x}, D_{y}\right)=\sum_{p=0}^{K} \sum_{q=0}^{L} \lambda_{p, q} D_{x}^{p} D_{y}^{q} & \\
& K, L>0, \lambda_{K, L} \neq 0
\end{aligned}
$$

is a 2-D linear PDO with real constant coefficients.

The functional (2.19) is a norm only in the trivial case that $L f=f$. This particular functional leads to the wellknown minimum norm solution of inverse problems [1]. Any other functional defined by (2.19) is a seminorm. This can easily be verified by showing that any nontrivial functional defined by (2.19) fails to satisfy the following property that characterizes any norm:

$$
\|L f\|>0 \quad \text { if } f \neq 0 .
$$

Obviously, there exists an $f^{*} \neq 0$ that satisfies the following partial differential equation $(P D E)$ :

$$
L\left(D_{x}, D_{y}\right) f(x, y)=0
$$

for which $\|L f\|=0$. In fact, any solution of the PDE (2.22) belongs to the null space of the functional (2.19), consisting of all $f \in C^{(K, L)}[R]$ for which $\|L f\|=0$.

The functionals defined by (2.19) can be seen as the direct extension into two variables of the one-variable functionals, whose minimization amounts to the so-called 1-D $L$-generalized splines [30]. Such functionals have been proposed in the formulation of the 1-D smoothing and interpolation problem [31]. The minimization of a functional of the form (2.19) was mentioned in [32] as the generalization of the $L$-generalized splines approach into 2-D interpolation. Some useful comments on the formulation proposed in [32] were reported by the authors in [33]. Finally, the minimization of certain functionals of the form (2.19) was successfully used by the authors in formulating the image interpolation problem [34].

Another useful class of nonnegative quadratic functionals is obtained by assuming that

$$
q_{k, l ; m, n}=\tau_{k, l} \delta_{k, m} \delta_{l, n}
$$

where $\tau_{k, l}$ are nonnegative real coefficients, and $\delta_{i, j}$ is the conventional Kronecker delta. Then, (2.10) amounts to the following class of nonnegative quadratic functionals:

$$
Q_{r}[f]=\iint\left(\sum_{p=0}^{K} \sum_{q=0}^{L} \tau_{p, q} f_{x^{p} y^{q}}^{2}\right) d x d y .
$$

These functionals can be seen as the direct extension into two variables of the corresponding one-variable functionals proposed by Tikhonov to be used as stabilizing functionals in 1-D problems [3]. A particular class of Tikhonov-type functionals, also known as spline functionals, was used in a variety of engineering applications [24]. A spline functional of order $M$ can be obtained from (2.24) if $M=K+L$ and

$$
\tau_{p, q}= \begin{cases}\left(\begin{array}{l}
M \\
p
\end{array}\right) & \text { if } p+q=M \\
0 & \text { if } p+q \neq M\end{cases}
$$

as follows:

$$
Q_{M}[f]=\iint\left(\sum_{p=0}^{M}\left(\begin{array}{c}
M \\
p
\end{array}\right)\left(\frac{\partial^{M} f}{\partial x^{p} \partial y^{M-p}}\right)^{2}\right) d x d y .
$$

Functionals of this type were used for interpolation of scattered data [35], [36]. In addition, such functionals appear very often in theory of elasticity, particularly in problems associated with a thin plate [37]. In particular, for $M=2$

$$
Q_{2}[f]=\iint\left(f_{x x}^{2}+2 f_{x y}^{2}+f_{y y}^{2}\right) d x d y
$$

This is the functional used by Grimson in the formulation of the visual surface reconstruction problem [19]. The use of this functional in this particular application was based on the proposed analogy between the smoothness of a visual surface and the equilibrium state of a thin plate [22].

\section{The Minimization Problem}

\section{A. Continuous Formulation}

Consider the continuous formulation of the image restoration problem based on the stabilizing functional approach. In general, the stabilizing functional can be any element of $\Phi_{S}$. According to Section II, the regularized solution is the function $f=f^{*} \in C^{(K, L)}[R]$ which minimizes $\Omega[f] \in \Phi_{S}$, under the constraint (2.1). Since the problem formulated is a conditional extremum problem, it can be solved by the method of Lagrange multipliers. That is, determine the $f=f_{\alpha}^{*} \in C^{(K, L)}[R]$ which minimizes the functional

$$
M_{\alpha}[f, g]=\|A f-g\|^{2}+\alpha \Omega[f]
$$

which is known as smoothing functional. The Lagrange parameter $\alpha$ is called here regularizing parameter. The equivalence between the minimization of (3.1) and the constrained minimization problem depends on the choice of $\alpha$. If the particular minimum $f=f_{\alpha}^{*}$ of the smoothing functional (3.1) also satisfies

$$
\left\|A f_{\alpha}^{*}-g\right\|=\delta
$$

the original constrained minimization problem is equivalent to the minimization of the smoothing functional (3.1). The necessary condition for the minimization of the 
smoothing functional (3.1) is given by the following theorem.

Theorem 2: Consider that a functional $\Omega[f] \in \Phi_{S}$ is used as a stabilizing functional. The necessary and sufficient condition that a function $f_{0} \in C_{0}^{(K, L)}[R]$ minimizes the smoothing functional $(3.1)$ is that it satisfies the following integro-differential equation:

$$
A^{*} A f+\alpha \Omega^{\prime}[f]=A^{*} g
$$

where $A^{*}$ is the adjoint operator for $A$, and $\Omega^{\prime}[f]$ is given by (2.7). dix B.

Proof: The proof of this theorem is given in Appen-

Theorem 2 can be the source of many regularized solutions of (1.1), depending on the specific choice of the stabilizing functional. However, the solution of (3.3) in practice is not a simple task. The analytical complexity of this problem is mainly determined by the form of the stabilizing functional chosen. For the sake of both analytical simplicity and statistical justification, this paper focuses on the constrained minimization of a quadratic stabilizing functional.

Suppose that a nonnegative quadratic functional $Q[f]$ of the form (2.10) is used as a stabilizing functional in the formation of the smoothing functional (3.1). It is shown in Appendix B that a function $f_{0} \in C_{0}^{(K, L)}[R]$ minimizes this smoothing functional if and only if it satisfies the following equation:

$$
\left(A^{*} A+\alpha B\right) f=A^{*} g
$$

where $B(\cdot)$ is the PDO defined by (2.13).

Suppose that a quadratic functional given by $(2.19)$ and (2.20) is used as a stabilizing functional. The necessary and sufficient condition for the minimization of the corresponding smoothing functional is given by (3.4), where

$$
B\left(D_{x}, D_{y}\right)=L^{*}\left(D_{x}, D_{y}\right) L\left(D_{x}, D_{y}\right)
$$

In (3.5), where $L(\cdot)$ is the PDO defined by (2.20), and $L^{*}(\cdot)$ is the adjoint operator for $L(\cdot)$ defined as follows:

$$
\begin{aligned}
L^{*}\left(D_{x}, D_{y}\right) & =L\left(-D_{x},-D_{y}\right) \\
& =\sum_{p=0}^{K} \sum_{q=0}^{L}(-1)^{p+q} \lambda_{p, q} D_{x}^{p} D_{y}^{q} .
\end{aligned}
$$

The operator $L^{*} L$ is called self-adjoint operator for $L(\cdot)$.

When a Tikhonov-type functional of the form (2.24) is used as a stabilizing functional, the $\operatorname{PDO} B(\cdot)$ is of the following form:

$$
B\left(D_{x}, D_{y}\right)=\sum_{k=0}^{K} \sum_{l=0}^{L}(-1)^{k+1} \tau_{k, l} D_{x}^{2 k} D_{y}^{2 l}
$$

Finally, the use of a spline functional of order $M$ as a stabilizing functional implies that

$$
B\left(D_{x}, D_{y}\right)=(-1)^{M}\left(D_{x}^{2}+D_{y}^{2}\right)^{M}
$$

In conclusion, the continuous formulation of the problem using a quadratic functional leads to the integro-differential equation (3.4). The particular quadratic functional used determines the form of this integro-differential equation. For a given quadratic stabilizing functional, the analytical treatment of (3.4) depends on the model describing the image formation process. Under the assumption that the image formation process can be described by a space-invariant model, (3.4) can be treated using Fourier transform techniques [38]. In general, the solution of (3.4) can be attempted using approximation methods. An obvious and rather simple approach is the discrete approximation of (3.4) using the finite difference method [39]. However, the solution of (3.4) using the finite difference method entails certain disadvantages. A qualitative evaluation of this method can be found in [23]. In this paper, the general problem is alternatively formulated directly in its discrete form.

\section{B. Discrete Formulation}

The problem of determining the discrete approximation of any quadratic functional of the form (2.10) is particularly complicated, even for a given finite difference approximation of the partial derivatives. However, the general form of the discrete approximation for this general class of functionals can be specified, regardless of the finite difference approximation used. In the analysis which follows, the form of this discrete approximation is determined using the finite element method, which is the most appropriate one for this type of problems [40]. A brief qualitative evaluation of the finite element method, as well as the mathematical arguments supporting its superiority over the finite difference method, can be found in [23].

Let $I_{R}$ be the image region, and consider its uniform separation by an operator $S$. Without loss of generality, consider that $S$ separates the image region $I_{R}$ into identical square elements $E$ of side $h$. Then any quadratic functional of the form (2.10) can be written as follows:

$$
Q[f]=\sum_{E \in S} \iint_{E}\left(\sum_{k, m=0}^{K} \sum_{l, n=0}^{L} f_{x^{k} y^{\prime}}^{E} q_{k, l ; m, n} f_{x^{m} y^{\prime \prime}}^{E}\right) d x d y
$$

Assuming that $(i, j)$ is the index for the upper-right-hand node of $E$, the quadratic functional (3.9) can be approximated as follows:

$$
Q^{d}[f]=\sum_{i=1}^{N} \sum_{j=1}^{N} \sum_{k, m=0}^{K} \sum_{l, n=0}^{L} f_{x^{k} y^{\prime}}(i, j) q_{k, l ; m, n} f_{x^{\prime \prime} y^{\prime \prime}}(i, j)
$$

where $f_{x^{m} y^{n}}(i, j) ; 1 \leq i, j \leq N$ denotes the approximation of $f_{x^{m} y^{n}}$ at the node $(i, j)$ in the image, obtained using a finite difference approximation as described in the analysis which follows. 
Consider the specific element $E$, whose upper righthand node corresponds to the image pixel indexed by ( $i$, j). Define

$$
f_{i, j}=f(i h, j h)
$$

Let $f(i, j)$ be the vector consisting of the image pixels surrounding the pixel indexed by $(i, j)$, defined as follows:

$$
f(i, j)=\left[\begin{array}{c}
f_{j}\left(i-m^{*}\right) \\
\vdots \\
f_{j}(i) \\
\vdots \\
f_{j}\left(i+m^{*}\right)
\end{array}\right], f_{j}(p)=\left[\begin{array}{c}
f_{p, j-n^{*}} \\
\vdots \\
f_{p, j} \\
\vdots \\
f_{p, j+n^{*}}
\end{array}\right] ;
$$

The integers $m^{*}, n^{*}$ are determined by the highest order of the derivative with respect to $x$ and $y$, respectively, appearing in the functional to be approximated. Using a finite difference approximation for the partial derivatives at $(i, j)$ node

$$
\begin{aligned}
f_{x^{m} y^{n}}(i, j)=\xi_{m, n}^{T} f(i, j) & \\
(m, n) & =(0,0), \cdots,(K, L)
\end{aligned}
$$

where $\xi_{m, n}$ is a vector of appropriate coefficients, depending on the specific finite difference approximation chosen. Substituting in (3.10) $f_{x^{m}, y^{n}}(i, j)$ from (3.13), the discrete approximation of $Q[f]$ becomes

$$
\begin{aligned}
Q^{d}[f]= & \sum_{i=1}^{N} \sum_{j=1}^{N} \sum_{k, m=0}^{K} \sum_{l, n=0}^{L} \\
& \cdot f(i, j)^{T} \xi_{k, l} q_{k . l: m, n} \xi_{m, n}^{T} f(i, j) \\
= & \sum_{i=1}^{N} \sum_{j=1}^{N} f(i, j)^{T} \boldsymbol{Q f}(i, j)
\end{aligned}
$$

where $Q$ is the following symmetric matrix:

$$
\boldsymbol{Q}=\sum_{k, m=0}^{K} \sum_{1, n=0}^{L} \xi_{k, l} q_{k, l: m, n} \xi_{m, n}^{T}
$$

Let $f$ be an $N^{2} \times 1$ vector whose elements are the lexicographically ordered elements of the image matrix. The vectors $f(i, j) ; 1 \leq i, j \leq N$ consist of elements lying inside the image region and, therefore, belonging to the image matrix, as well as elements lying outside the image region, called boundary elements. To separate the boundary elements from the elements of the image matrix, de- fine the following set:

$$
\begin{aligned}
& S_{m^{*} n^{*}}(f)=\left\{s p\left\{f_{1-m^{*}, j} ; f_{N+m^{*}, j} ; f_{i, 1-n^{*}} ; f_{i, N+n^{*}}\right\}:\right. \\
& 1-m^{*} \leq i \leq N+m^{*}, \\
&\left.1-n^{*} \leq j \leq N+n^{*}\right\}
\end{aligned}
$$

where $s p\left\{z_{1} ; z_{2} ; \cdots ; z_{L}\right\}$ denotes the linear span of the variables $z_{i} ; i=1, \cdots, L$. Now consider the $N^{2} \times 1$ vector $f^{\prime}$ whose elements $f_{i}^{\prime} ; i=1, \cdots, N^{2}$ belong to the set $S_{m^{*} n^{*}}(f)$, that is,

$$
f_{i}^{\prime} \in S_{m^{*} n^{*}}(f) \forall i=1, \cdots, N^{2} .
$$

Taking into account the above definitions, there always exists an $N^{2} \times N^{2}$ matrix $\boldsymbol{B}$, such that

$$
Q^{d}[f]=\sum_{i=1}^{N} \sum_{j=1}^{N} \boldsymbol{f}(i, j)^{T} \boldsymbol{Q} \boldsymbol{f}(i, j)=\boldsymbol{f}^{T} \boldsymbol{B} \boldsymbol{f}+\boldsymbol{B}^{\prime} \boldsymbol{f}^{\prime}
$$

where $\boldsymbol{B}^{\prime}$ is an $N^{2} \times N^{2}$ matrix whose elements $b_{i, j}^{\prime} ; 1 \leq$ $i, j \leq N^{2}$ are formed as linear combinations of the boundary elements, that is, $b_{i, j}^{\prime} \in S_{m^{*} n^{*}}(f)$. In practice, the highest order of the partial derivatives appearing in quadratic functionals used is a small integer; thus, the effect of the boundary elements on the approximation can be neglected. Under this assumption, the discrete approximation of $Q[\cdot]$ becomes

$$
Q^{d}[f]=\langle\boldsymbol{f}, \boldsymbol{B} \boldsymbol{f}\rangle
$$

where $f$ is an $N^{2} \times 1$ vector, and $\boldsymbol{B}$ is a symmetric $N^{2} \times$ $N^{2}$ matrix, depending on the specific quadratic functional considered and the finite difference approximation used. In fact, the condition that guarantees the validity of (3.18) is the following:

$$
\boldsymbol{B}^{\prime} \boldsymbol{f}^{\prime}=0
$$

This boundary condition is related to the assumption that $f \in C_{0}^{(K, L)}[R]$, which guarantees the validity of the representation of a continuous quadratic functional by (2.14).

The discrete approximation of the class of quadratic functionals defined by (2.19) is of particular interest, owing to the existence of a unique correspondence between any functional given by (2.19) and a certain PDE. Given the discrete approximation of this PDE, the discrete approximation of its corresponding functional can be explicitly determined, as described by the following proposition.

Proposition 1: Consider any quadratic functional given by the combination of (2.19) and (2.20). Furthermore, consider the corresponding PDE

$$
L\left(D_{x}, D_{y}\right) f(x, y)=u(x, y)
$$

and let its discrete approximation, obtained using a finite difference approximation of the partial derivatives, be the following:

$$
\boldsymbol{L} \boldsymbol{f}=\boldsymbol{u}
$$


where $f$ is the $N^{2} \times 1$ vector defined above, and $L$ is an $N^{2} \times N^{2}$ matrix, depending on the specific PDO and the finite difference approximation used. The discrete approximation of the functional $Q_{L}[\cdot]$, obtained using the finite element method and the same finite difference approximation of the partial derivatives, is given by

$$
Q_{L}^{d}[f]=\|\boldsymbol{L} f\|^{2}
$$

where $\boldsymbol{L}$ is the matrix appearing in (3.21).

Proof: The proof of this proposition is given in Appendix C.

The discrete approximation of the class of quadratic functionals defined by (2.19) can also be written as follows:

$$
Q_{L}^{d}[f]=\|\boldsymbol{L f}\|^{2}=\langle\boldsymbol{L f}, \boldsymbol{L f}\rangle=\left\langle\boldsymbol{f}, \boldsymbol{L}^{T} \boldsymbol{L} \boldsymbol{f}\right\rangle .
$$

Therefore, the discrete approximation of any quadratic functional defined by (2.19) can be seen as the special case of the approximation given by (3.18) for which

$$
\boldsymbol{B}=\boldsymbol{L}^{T} \boldsymbol{L}
$$

The discrete formulation of the image restoration problem on the basis of the stabilizing functional approach is now presented. The image formation is described in this case by the discrete model (1.3). In addition, it is assumed that an admissible quadratic functional of the form (2.10) is used as a stabilizing functional. On the basis of the analysis presented above, the problem of obtaining regularized solutions of (1.3) can be formulated as follows. Determine $f \in R^{N^{2}}$ such that

$$
\langle\boldsymbol{f}, \boldsymbol{B} \boldsymbol{f}\rangle \rightarrow \min
$$

under the constraint that

$$
\|\boldsymbol{A} \boldsymbol{f}-\boldsymbol{g}\|=\delta
$$

where $\delta$ is a positive constant. Using the method of Lagrange multipliers, the above problem amounts to the minimization of the following quadratic form:

$$
M_{\alpha}^{d}[\boldsymbol{f}, \boldsymbol{g}]=\|\boldsymbol{A} \boldsymbol{f}-\boldsymbol{g}\|^{2}+\alpha\langle\boldsymbol{f}, \boldsymbol{B} \boldsymbol{f}\rangle
$$

where $\langle\cdot, \cdot\rangle:, R^{N^{2}} \times R^{N^{2}} \rightarrow R$ is the Euclidean inner product and $\|\cdot\|$ is the corresponding norm.

The necessary and sufficient condition for the minimization of (3.27) is now presented [29]. Consider the quadratic form (3.27), where $\boldsymbol{B}$ is a nonnegative definite, real symmetric matrix. The necessary and sufficient condition that $f=f_{\alpha}^{*} \in R^{N^{2}}$ minimizes the quadratic form (3.27) is that $f_{\alpha}^{*}$ satisfies the following set of linear equations:

$$
\boldsymbol{A}_{R} f=\boldsymbol{A}^{T} \boldsymbol{g}
$$

where

$$
\boldsymbol{A}_{R}=\boldsymbol{A}^{T} \boldsymbol{A}+\alpha \boldsymbol{B} .
$$

In the particular case that the stabilizing functional considered belongs to the class of quadratic functionals defined by (2.19), the quadratic form to be minimized is the following:

$$
M_{\alpha}^{d}[\boldsymbol{f}, \boldsymbol{g}]=\|\boldsymbol{A} \boldsymbol{f}-\boldsymbol{g}\|^{2}+\alpha\|\boldsymbol{L} \boldsymbol{f}\|^{2} .
$$

Taking into account (3.24), the necessary condition for the minimization of (3.30) can be directly obtained as a special case of the above minimization problem. The necessary and sufficient condition that $f=f_{\alpha}^{*} \in R^{N^{2}}$ minimizes the quadratic form (3.30) is that $f_{\alpha}^{*}$ satisfies the set of linear equations (3.28), where

$$
\boldsymbol{A}_{R}=\boldsymbol{A}^{T} \boldsymbol{A}+\alpha \boldsymbol{L}^{T} \boldsymbol{L} .
$$

This particular formulation of the problem is known in the literature as constrained least squares image restoration [9], [41], [42]. This formulation of the problem defines a very broad class of solutions to the image restoration problem, in the sense that for any $L$ the minimization of (3.30) amounts to a different solution. On the other hand, the minimization problem resulting from the previous analysis can also be obtained by formulating the problem in a different way. In fact, the minimization of the quadratic form given by (3.30) is the result of the following formulation [8], [42]:

$$
\|A f-g\| \leq \epsilon
$$

and

$$
\|\boldsymbol{L} \boldsymbol{f}\| \leq E
$$

where $E$ is a "fixed" number and $\epsilon$ is a "small" number. The only information existing about $L$, called "constraint operator" in this approach, is that it is a bounded linear operator. The approach itself, however, neither provides information as to what operator $\boldsymbol{L}$ should be, nor of a general procedure to form such operators. In most of the approaches which have appeared to date, $L$ is an operator to be chosen. Although several operators $\boldsymbol{L}$ have been proposed, the problem of choosing such operators in a physically meaningful manner still remains an open one. According to the formulation proposed here, the operator $\boldsymbol{L}$ is clearly related to the minimization of the quadratic functional defined by (2.19), whose discrete approximation is given by (3.22). The physical meaning of choosing a specific functional to be minimized, or equivalently an operator $L$ in (3.30), is investigated in the sequel.

\section{Regularized Solutions Based on MaP ESTIMATION}

This section presents the MAP estimation interpretation for a class of regularized solutions based on the stabilizing functional approach. The analysis which follows relates the stabilizing functional used in the minimization problem with the description of the a priori knowledge regarding the original image [43]. The importance of this analysis is twofold. First, it provides a stochastic interpretation for the entire family of constrained least squares approaches which have appeared in the literature. On the other hand, it constructs the necessary background for the development of a variety of regularized solutions reflecting the stochastic nature of the image restoration problem.

The concept of the MAP estimation has already been used in an attempt to solve the image restoration problem [1]. There exist two main points which can be underlined 
about the existing MAP estimation approaches in image restoration. In terms of the formulation of the MAP estimation problem followed, the key issue is the description of the a priori knowledge assumed for the original object. In terms of the algorithms produced, the key issue is the statistical information required for the object and the noise.

Let $f$ be the object to be estimated and $g$ the observed data, i.e., the recorded image. The MAP estimate of the object is defined [44] as that $f=\hat{f}_{\mathrm{MAP}}$ for which

$$
\ln p(g / f)+\ln p(f) \rightarrow \max
$$

where $p(g / f)$ is the pdf for the data $g$ given the original object and $p(f)$ is the pdf of the object. In (4.1), In $p(g / f)$ expresses the probabilistic dependence of $g$ on $f$, while $\ln p(f)$ is the a priori knowledge regarding the original object.

The description of the a priori knowledge regarding the original object is very crucial in the MAP estimation approach [43]. The specific pdf of the object chosen determines the statistical information that is necessary for the implementation of the resulting MAP restoration algorithm. The pdf which is frequently used for the description of the a priori knowledge existing for the object is the following [1]:

$$
p(f)=k \exp \left\{-\frac{1}{2}(f-\bar{f})^{T} \Lambda^{-1}(f-\bar{f})\right\}
$$

where $\bar{f}$ is the mean and $\Lambda$ is the covariance matrix of the object $f$. When the a priori knowledge regarding the original object is described by (4.2), the second-order statistics of the object are assumed to be known. However, this assumption is far away from the reality in most practical situations.

Jain, looking beyond the conventional covariance models used to approximate the second-order statistics of the image, proposed in [45] that the images can be represented by SPDE image models. Without entering into a detailed discussion, the general concept of image representation using SPDE models is briefly described here. Consider the following PDE:

$$
L\left(D_{x}, D_{y}\right) u(x, y)=v(x, y)
$$

where $L(\cdot)$ is the PDO defined in (2.20) and $v(\cdot)$ is a specified function. On the basis of the necessary condition for any PDE given by (4.3) to be a well-posed Cauchy problem [45], the PDE's are distinguished into three main categories; namely, hyperbolic, parabolic, and elliptic PDE's. Reference [45] established the correspondence between the three above categories of PDE's and the nature of the image representation in terms of causality. Consider now the following SPDE:

$$
L\left(D_{x}, D_{y}\right) f(x, y)=\epsilon(x, y)
$$

where $L(\cdot)$ is, in general, a 2-D linear PDO defined in (2.20), $f(\cdot)$ is a process defined in a 2-D random field which represents the image, and $\epsilon(\cdot)$ is a white noise process defined in a $2-\mathrm{D}$ random field as well. It was
TABLE 1

Stochastic Partial Differential Equation Image Models

\begin{tabular}{l|c|c|c}
\hline \multicolumn{1}{c|}{ SPDE } & $\begin{array}{c}\text { Partial Differential } \\
\text { Operator } \\
\text { Image Model }\end{array}$ & Kind of & Comments \\
\hline Causal 1 (C1) & $\left(D_{x}, D_{y}\right)$ & PDE & on PDEs \\
\hline $\begin{array}{l}\text { Semicausal } \\
\text { (SC1) }\end{array}$ & $\left.D_{y}+\alpha_{2}\right)\left(D_{y}+\alpha_{1}\right)$ & Hyperbolic & $\begin{array}{l}\text { Hyperbolic Wave } \\
\text { Equation }\end{array}$ \\
\hline $\begin{array}{l}\text { Semicausal } \\
\text { (SC2) }\end{array}$ & $\left(D_{x}{ }^{2}+\alpha_{1}\right)\left(D_{y}+\alpha_{2}\right)$ & Parabolic & $\begin{array}{l}\text { Separable Para- } \\
\text { bolic Equation }\end{array}$ \\
\hline $\begin{array}{l}\text { Noncausal 1 (NC1) } \\
\text { Noncausal 2 (NC2) }\end{array}$ & $D_{x}{ }^{2}+D_{y}{ }^{2}+\alpha$ & Elliptic & $\begin{array}{l}\text { Parabolic Diffu- } \\
\text { sion Equation Equation }\end{array}$ \\
\hline Noncausal 3 (NC3) & $\left(D_{x}{ }^{2}+D_{y}{ }^{2}\right)^{2}$ & Elliptic & $\begin{array}{l}\text { Biharmonic Equa- } \\
\text { tion }\end{array}$ \\
\hline
\end{tabular}

shown in [45] that the SPDE image model given by (4.4) corresponds to a causal, semicausal, or noncausal image representation, given that the corresponding PDO defines a hyperbolic, parabolic, or elliptic PDE, respectively. Certain SPDE image models were also proposed in [45] and evaluated in terms of their performance in representing different kinds of images. These models are presented in Table $\mathrm{I}$.

In image restoration, it is commonly considered that the recorded image is contaminated by additive noise due to the image formation system, that is,

$$
n=A f-g \text {. }
$$

Given that the object $f$ and the degradation operator $A$ are fixed, the conditional pdf of the recorded image $g$ given the original object $f$ can be equivalently expressed as the pdf of the noise process $n$ associated with the image formation system. Assuming that the noise due to the image formation system is a white Gaussian process with variance $\sigma_{n}^{2}, p(g / f)$ is given by

$$
\begin{aligned}
p(g / f) & =p(n)=k_{1} \exp \left\{-\frac{\|n\|^{2}}{2 \sigma_{n}^{2}}\right\} \\
& =k_{1} \exp \left\{-\frac{\|A f-g\|^{2}}{2 \sigma_{n}^{2}}\right\}
\end{aligned}
$$

where $k_{1}$ is a positive constant depending on $\sigma_{n}$.

Assume now that the a priori knowledge existing for the image is that it can be described by the SPDE model given in (4.4). Assume, in addition, that the white noise process $\epsilon(\cdot)$ is also Gaussian with variance $\sigma_{\epsilon}^{2}$. Given the PDO $L(\cdot)$ used for the representation of the image, the image process $f(\cdot)$ is uniquely related with the noise process $\epsilon(\cdot)$ by (4.4). Therefore, under this assumption, the pdf $p(f)$ of the image is in fact the pdf of the noise process $\epsilon(\cdot)$ associated with this specific image representa- 
tion. Taking into account the assumption that $\epsilon(\cdot)$ is a Gaussian white noise process

$$
\begin{aligned}
p(f) & =p(\epsilon)=k_{2} \exp \left\{-\frac{\|\epsilon\|^{2}}{2 \sigma_{\epsilon}^{2}}\right\} \\
& =k_{2} \exp \left\{-\frac{\|L f\|^{2}}{2 \sigma_{\epsilon}^{2}}\right\} .
\end{aligned}
$$

Combining (4.6) and (4.7) with (4.1), it can easily be shown that the MAP estimate is the particular $\hat{f}_{\text {MAP }} \in$ $C^{(K, L)}[R]$ which minimizes the following functional:

$$
M_{\alpha}[f, g]=\|A f-g\|^{2}+\alpha\|L f\|^{2}
$$

where $\alpha$ is a positive constant given by

$$
\alpha=\frac{\sigma_{n}^{2}}{\sigma_{\epsilon}^{2}}
$$

In the discrete case, the MAP estimation interpretation is based on the assumption that the image can be represented by the discrete approximation of a certain SPDE image model, given by

$$
\boldsymbol{L} \boldsymbol{f}=\boldsymbol{\varepsilon}
$$

where $f$ is the image vector, $L$ is a model dependent matrix, and $\varepsilon$ is a vector consisting of zero-mean white noise elements. Following a similar reasoning (see also [43]), it can be shown that the MAP estimate is the $\hat{f}_{\mathrm{MAP}} \in \boldsymbol{R}^{N^{2}}$ which minimizes the following quadratic form:

$$
M_{\alpha}^{d}[\boldsymbol{f}, \boldsymbol{g}]=\|\boldsymbol{A} \boldsymbol{f}-\boldsymbol{g}\|^{2}+\alpha\|\boldsymbol{L f}\|^{2} .
$$

In conclusion, given that the a priori knowledge about the image can be described by an SPDE image model, the MAP estimate of the object is the result of the minimization problem (4.8) or (4.11). This is exactly the minimization problem developed on the basis of the stabilizing functional approach, given that the stabilizing functional used belongs to the class of stabilizing functionals defined by (2.19). The MAP interpretation of the stabilizing functional approach can therefore be described as follows. Given that the stabilizing functional used is based on a certain PDO, the regularized solution obtained is the MAP estimate of the object assuming that the $a$ priori knowledge for the image can be described by the corresponding SPDE image model. Conversely, given that an image can be represented by a certain SPDE model, the MAP estimate of the object is the result of the minimization problem (4.8) or (4.11).

According to this analysis, there exists a one-to-one correspondence between the image representation by an SPDE model assumed and the stabilizing functional to be chosen in the minimization problem. In other words, the problem of selecting the "optimum" stabilizing functional for a given application is equivalent with the problem of determining the "optimum" SPDE representation for the given original image, or the class of images. On the other hand, the above interpretation provides a link between MAP estimation and constrained least squares in image restoration. In fact, any constraint operator proposed can be related to the description of the a priori knowledge existing for the image by the discrete approximation of a certain SPDE image model. In this paper, a number of models proposed in [45] to represent images are considered to be the source of constraint operators.

\section{Regularizing Filters}

This section presents the derivation of a variety of filters resulting from the application of RT in continuous image restoration. In general, the image formation process is described by the space-variant model (1.2). To simplify the solution of the integro-differential equation (3.4), it is now assumed that the image formation process can be described by a space-invariant model. Fortunately, this assumption is realistic in many image restoration applications. The physical meaning of such an assumption is that the image formation process has a uniform effect on both the object and the image plane. The direct analytical implication of the above assumption is that the PSF is a function of the differences between the variables belonging to the image and the object plane. In the case of space-invariant formulation, the image formation process is described by the following equation:

$$
\begin{gathered}
\iint h(u-x, v-y) f(x, y) d x d y+n(u, v) \\
=g(u, v) .
\end{gathered}
$$

In this case, the image formation is described by $2-\mathrm{D}$ convolution. Therefore, image restoration corresponds to deconvolution, well known as ill-posed inverse problem in the literature.

Suppose that a nonnegative quadratic functional of the form (2.10) is used as a stabilizing functional. The necessary and sufficient condition for the minimization of the corresponding smoothing functional is obtained from (3.4) as follows:

$$
\begin{gathered}
\iint \bar{h}(x-u, y-v) f(u, v) d u d v+\alpha B\left(D_{x}, D_{y}\right) f(x, y) \\
=\bar{g}(x, y)
\end{gathered}
$$

where

$$
\begin{aligned}
& \bar{h}(x, y)=\iint h(\xi-x, \zeta-y) h(\xi, \zeta) d \xi d \zeta \\
& \bar{g}(x, y)=\iint h(\xi-x, \zeta-y) g(\xi, \zeta) d \xi d \zeta
\end{aligned}
$$

and $B(\cdot)$ is the PDO defined by (2.13).

It is proven in Appendix $D$ that the solution of the integro-differential equation of the form (5.2) can be obtained by passing the data through a filter, called regularizing filter $(R F)$. For a given PDO $B(\cdot)$, the transfer function of this filter is given by

$$
H_{R}\left(\omega_{1}, \omega_{2}, \alpha\right)=\frac{H^{*}\left(\omega_{1}, \omega_{2}\right)}{\left|H\left(\omega_{1}, \omega_{2}\right)\right|^{2}+\alpha R\left(\omega_{1}, \omega_{2}\right)}
$$


where $R(\cdot)$ is a polynomial, called regularizing polynomial, defined uniquely in terms of the quadratic functional considered by

$$
R\left(\omega_{1}, \omega_{2}\right)=B\left(j \omega_{1}, j \omega_{2}\right) .
$$

The parameter $\alpha$, which is the Lagrange multiplier in the minimization problem formulated, is called regularizing parameter. When a quadratic functional given by (2.19) and $(2.20)$ is used as a stabilizing functional

$$
R\left(\omega_{1}, \omega_{2}\right)=\left|L\left(j \omega_{1}, j \omega_{2}\right)\right|^{2}
$$

Table II presents a variety of regularizing polynomials based on the SPDE image models proposed in [45]. When a Tikhonov-type quadratic functional of the form (2.24) is used as a stabilizing functional

$$
R\left(\omega_{1}, \omega_{2}\right)=\sum_{k=0}^{K} \sum_{l=0}^{L} \tau_{k, l} \omega_{l}^{2 k} \omega_{2}^{2 l}
$$

Finally, the use of a spline functional of order $M$ as a stabilizing functional amounts to

$$
R\left(\omega_{1}, \omega_{2}\right)=\left(\omega_{1}^{2}+\omega_{2}^{2}\right)^{M} .
$$

By its definition, the regularizing polynomial is nonnegative definite, i.e.,

$$
R\left(\omega_{1}, \omega_{2}\right) \geq 0 \forall \omega_{1}, \omega_{2} \in(-\infty,+\infty) .
$$

The nonnegative definition of all the regularizing polynomials obtained is closely related to the nonnegativeness of the quadratic stabilizing functionals and, therefore, to the existence of a minimum for this class of functionals considered. The transfer function given by $(5.5)$ depends on the specific PDO chosen. In this sense, (5.5) determines a very broad class of regularizing filters. Since the relation between the PDO $B(\cdot)$ and the regularizing polynomial $R(\cdot)$ is unique, there is one-to-one correspondence between any quadratic stabilizing functional chosen, and the RF obtained [38].

The RF defined by (5.5) can also be written as follows:

$$
H_{R}\left(\omega_{1}, \omega_{2}, \alpha\right)=\frac{P\left(\omega_{1}, \omega_{2}, \alpha\right)}{H\left(\omega_{1}, \omega_{2}\right)}
$$

where

$$
P\left(\omega_{1}, \omega_{2}, \alpha\right)=\frac{\left|H\left(\omega_{1}, \omega_{2}\right)\right|^{2}}{\left|H\left(\omega_{1}, \omega_{2}\right)\right|^{2}+\alpha R\left(\omega_{1}, \omega_{2}\right)} .
$$

Clearly, the effect of regularization is carried by the function $P(\cdot)$, defined by $(5.12)$. This is an indication that the generalization of the filter $(5.11)$ can be achieved by determining a more general class of functions $P(\cdot)$ in such a way that the corresponding filter defined by (5.11) amounts to regularized solutions of the image restoration problem. The existence of such functions was proven in

\begin{tabular}{|c|c|c|}
\hline $\begin{array}{c}\text { SPDE } \\
\text { Image Model }\end{array}$ & $\begin{array}{l}\text { Partial Differential } \\
\text { Operator } \\
\qquad L\left(D_{x}, D_{y}\right)\end{array}$ & $\begin{array}{l}\text { Regularizing Polyno- } \\
\text { mial } \\
\qquad R\left(\omega_{1}, \omega_{2}\right)\end{array}$ \\
\hline Causal I (Cl) & $\left(D_{x}+\alpha_{2}\right)\left(D_{y}+\alpha_{1}\right)$ & $\left(\omega_{1}^{2}+\alpha_{2}^{2}\right)\left(\omega_{2}^{2}+\alpha_{1}^{2}\right)$ \\
\hline Semicausal 1 (SC1) & $D_{y}-D_{x}^{2}+\alpha$ & $\omega_{2}^{2}+\left(\omega_{1}^{2}+\alpha\right)^{2}$ \\
\hline Semicausal 2 (SC2) & $\left(D_{x}^{2}+\alpha_{1}\right)\left(D_{y}+\alpha_{2}\right)$ & $\left(\omega_{1}^{2}-\alpha_{1}\right)^{2}\left(\omega_{2}^{2}+\alpha_{2}^{2}\right)$ \\
\hline Noncausal $1(\mathrm{NC} 1)$ & $D_{x}^{2}+D_{y}^{2}+\alpha$ & $\left(\omega_{1}^{2}+\omega_{2}^{2}-\alpha\right)^{2}$ \\
\hline Noncausal 2 (NC2) & $\left(D_{x}^{2}+D_{y}^{2}\right)^{2}$ & $\left(\omega_{1}{ }^{2}+\omega_{2}{ }^{2}\right)^{4}$ \\
\hline Noncausal 3 (NC3) & $\left(D_{x}^{2}+\alpha\right)\left(D_{y}^{2}+\alpha\right)$ & $\left(\omega_{1}^{2}-\alpha\right)^{2}\left(\omega_{2}^{2}-\alpha\right)^{2}$ \\
\hline
\end{tabular}
[29] by extending the concept of the RO into 2-D problems. Some elements of this analysis were reported by the authors in [46]. According to this analysis, any filter of the form (5.11) amounts to regularized solutions of the
TABLE II

Regularizing Polynomials Based on SPDE Image Models

image restoration problem, provided that the corresponding function $P(\cdot)$ satisfies the following conditions:

a) $P\left(\omega_{1}, \omega_{2}, \alpha\right)$ is defined on the region $\{\alpha \geq 0,-\infty$ $\left.<\omega_{1}, \omega_{2}<+\infty\right\}$.

b) For every $\alpha \geq 0,0 \leq P\left(\omega_{1}, \omega_{2}, \alpha\right) \leq 1$.

c) $P\left(\omega_{1}, \omega_{2}, 0\right)=1$.

d) For every $\alpha>0$ and $-\infty<\omega_{1}, \omega_{2}<+\infty, P\left(\omega_{1}\right.$, $\left.\omega_{2}, \alpha\right)=P\left(-\omega_{1},-\omega_{2}, \alpha\right)$

e) For every $\alpha>0, P\left(\omega_{1}, \omega_{2}, \alpha\right) \rightarrow 0$ when $\omega_{1}, \omega_{2}$ $\rightarrow \infty$.

f) $P\left(\omega_{1}, \omega_{2}, \alpha\right) \rightarrow 1$ nondecreasingly as $\alpha \rightarrow 0$, and this convergence is uniform on every interval $\left|\omega_{i}\right|$ $\leq \omega_{c},(i=1,2)$.

g) For every $\alpha>0$,

$$
\frac{P\left(\omega_{1}, \omega_{2}, \alpha\right)}{H\left(\omega_{1}, \omega_{2}\right)} \in L_{2}(-\infty,+\infty) .
$$

h) For every $\omega_{i} \neq 0(i=1,2), P\left(\omega_{1}, \omega_{2}, \alpha\right) \rightarrow 0$, when $\alpha \rightarrow \infty$, and this convergence is uniform on every interval of the form $\left[\omega_{i 1}, \omega_{c_{2}}\right]$ where $0<\omega_{(1)}$ $<\omega_{c 2}$.

In conclusion, given that the image formation process can be described by a space-invariant model, any function $P(\cdot)$ satisfying the above conditions determines a filter with transfer function given by (5.11). With the observed data being the input of such a filter, the output is a regularized solution to (5.1). Therefore, the problem of developing alternative regularized solutions to $(5.1)$ leads to the equivalent problem of appropriately selecting the functions $P(\cdot)$.

Consider the following function:

$$
P\left(\omega_{1}, \omega_{2}, \alpha\right)=\left[\frac{\left|H\left(\omega_{1}, \omega_{2}\right)\right|^{2}}{\left|H\left(\omega_{1}, \omega_{2}\right)\right|^{2}+\alpha R\left(\omega_{1}, \omega_{2}\right)}\right]^{\gamma}
$$

where $\gamma \in(0,1], \alpha>0$ and $R(\cdot)$ is a polynomial which satisfies the following conditions:

a) It is piecewise continuous on every finite interval. 
b) It is nonnegative and, in addition, $R\left(\omega_{1}, \omega_{2}\right)>0 \forall$ $\omega_{1}, \omega_{2} \neq 0$.

c) It is symmetric with respect to the origin, that is, $R\left(\omega_{1}, \omega_{2}\right)=R\left(-\omega_{1},-\omega_{2}\right)$.

d) $R\left(\omega_{1}, \omega_{2}\right) \geq M>0$ for sufficiently large $\left|\omega_{i}\right|$; $i=1,2$.

It can easily be verified that any function defined by (5.13) satisfies the above conditions, provided that

$$
\begin{aligned}
& {\left[\frac{1}{H\left(\omega_{1}, \omega_{2}\right)}\right]^{1-\gamma}\left[\frac{H^{*}\left(\omega_{1}, \omega_{2}\right)}{\left|H\left(\omega_{1}, \omega_{2}\right)\right|^{2}+\alpha R\left(\omega_{1}, \omega_{2}\right)}\right]^{\gamma}} \\
& \quad \in L_{2}(-\infty,+\infty) .
\end{aligned}
$$

Having already introduced the class of functions $P(\cdot)$ described by (5.13), a class of regularizing filters can be subsequently defined. The transfer function of this class of filters is obtained by combining (5.11) and (5.13) as follows:

$$
\begin{aligned}
& H_{R}\left(\omega_{1}, \omega_{2}, \alpha\right) \\
& \quad=\left[\frac{1}{H\left(\omega_{1}, \omega_{2}\right)}\right]^{1-\gamma}\left[\frac{H^{*}\left(\omega_{1}, \omega_{2}\right)}{\left|H\left(\omega_{1}, \omega_{2}\right)\right|^{2}+\alpha R\left(\omega_{1}, \omega_{2}\right)}\right]^{\gamma} .
\end{aligned}
$$

The filters defined by (5.15) are called generalized regularizing filters (GRF's).

The class of GRF's defined by (5.15) consists of an infinite number of elements for a given combination of $\alpha$ and $R(\cdot)$ and for all $\gamma \in(0,1)$. By definition, the value $\gamma=0$ is not an allowable one for the free parameter $\gamma$. In such a case, (5.13) amounts to $P\left(\omega_{1}, \omega_{2}, \alpha\right)=1$. Therefore, $\gamma=0$ is the limiting and, in fact, trivial case where there is no regularization applied on the derivation of the solution. Not surprisingly, in this case, the transfer function given by (5.15) amounts to the transfer function of the ill-behaved inverse filter. The case of $\gamma=1$ is also of special interest here. In this case, the class of GRF's defined by (5.15) amounts to (5.5), which is exactly the class of RF's derived on the basis of the stabilizing functional approach. Under this consideration, the stabilizing functional approach can be seen as a special methodology in constructing RO's.

Among all values of $\gamma \in(0,1)$, the specific value $\gamma=\frac{1}{2}$ is of special interest. In this case, the transfer function given by (5.15) amounts to

$$
\begin{aligned}
& H_{R}\left(\omega_{1}, \omega_{2}, \alpha\right) \\
& =\left[\frac{H^{*}\left(\omega_{1}, \omega_{2}\right)}{H\left(\omega_{1}, \omega_{2}\right)}\right]^{1 / 2}\left[\frac{1}{\left|H\left(\omega_{1}, \omega_{2}\right)\right|^{2}+\alpha R\left(\omega_{1}, \omega_{2}\right)}\right]^{1 / 2} .
\end{aligned}
$$

If, in addition, $H^{*}\left(\omega_{1}, \omega_{2}\right)=H\left(\omega_{1}, \omega_{2}\right)$, which is the case in most of the image formation systems, then

$$
H_{R}\left(\omega_{1}, \omega_{2}, \alpha\right)=\left[\frac{1}{\left|H\left(\omega_{1}, \omega_{2}\right)\right|^{2}+\alpha R\left(\omega_{1}, \omega_{2}\right)}\right]^{1 / 2} .
$$

\section{Vi. Regularized Solutions in Discrete Image REstoration}

\section{A. Regularized Solutions Based on Noncausal SPDE Image Models}

Many of the early image restoration approaches based on constrained optimization were motivated by the use of cubic splines in 2-D problems [1], [2], [7]. The resulting algorithms were based on two 1-D cubic splines, each one corresponding to the horizontal and vertical directions. The relationship between cubic splines and the minimization of a quadratic functional is now investigated. It is well known that the 1-D cubic splines result from the minimization of the following functional of one variable:

$$
\int\left(D_{x}^{2} f\right)^{2} d x=\int\left(\frac{d^{2} f}{d x^{2}}\right)^{2} d x
$$

The direct generalization of the functional (6.1) amounts to the following functional of two variables:

$$
\iint\left(D_{x}^{2} D_{y}^{2} f\right)^{2} d x d y=\iint\left(\frac{\partial^{2}}{\partial x^{2}} \frac{\partial^{2}}{\partial y^{2}} f\right)^{2} d x d y
$$

This is the functional chosen to be minimized in [7]. Obviously, the functional defined by (6.2) is a special case of the class of quadratic functionals defined by (2.19), corresponding to a separable, elliptic PDO $L(\cdot)$.

The interpretation traditionally associated with quadratic functionals is that they control the smoothness of the resulting image. However, on the basis of the analysis presented in Section IV, their interpretation can be more elaborate and selective. In fact, the use of a separable quadratic functional of the form (6.2) implies the representation of the image by a separable noncausal SPDE image model. This statement determines the limited efficiency of such functionals, given the limitation of separable SPDE image models in representing images. In fact, it has been shown [45] that the nonseparable SPDE image models are more suitable in representing real world images. Several operators corresponding to noncausal models are presented here [45].

The discrete approximation of any noncausal SPDE image model can be written in a matrix form as follows:

$$
\sum_{l=1}^{L} \boldsymbol{P}_{l} \boldsymbol{F}_{n} \boldsymbol{Q}_{l}=\boldsymbol{E}_{n}
$$

where $\boldsymbol{E}_{n}$ is an $N \times N$ matrix consisting of zero-mean white noise elements, and $\boldsymbol{F}_{n}$ denotes the original $N \times N$ image matrix $\boldsymbol{F}$. The subscript " $n$ " is used here to indicate that $(6.3)$ is the discrete approximation of a noncausal image model. In general, $\boldsymbol{P}_{l}$ and $\boldsymbol{Q}_{l} ; l=1, \cdots$, $L$ are $N \times N$ symmetric, $m$-diagonal, Toeplitz matrices which depend on the specific SPDE image model considered and the finite difference approximation used in obtaining its discrete approximation. Assuming that $f_{n}\left(\varepsilon_{n}\right)$ 
is the $N^{2} \times 1$ vector consisting of the lexicographically ordered elements of the matrix $\boldsymbol{F}_{n}\left(\boldsymbol{E}_{n}\right)$, (6.3) can be equivalently written as

$$
L_{n} f_{n}=\varepsilon_{n}
$$

where

$$
\boldsymbol{L}_{n}=\sum_{l=1}^{L} \boldsymbol{P}_{l} \otimes \boldsymbol{Q}_{l}
$$

while $\otimes$ denotes the Kronecker product between matrices.

Among noncausal SPDE image models, the separable ones possess the simplest discrete approximation. In fact, in the case of separable noncausal SPDE models, $\boldsymbol{L}_{n}$ consists of only one term. The discrete approximation of various noncausal SPDE image models is presented in Table III [45]. In this approximation, $I$ is the $N \times N$ identity matrix, and $Q$ is a tridiagonal, symmetric, Toeplitz matrix whose elements are given by

$$
q_{i j}=\left\{\begin{array}{cl}
q_{0} & \text { if } i=j \\
-q_{1} & \text { if }|i-j|=1 \\
0 & \text { otherwise }
\end{array}\right.
$$

where $q_{0}=\frac{1}{2}$ and $q_{1}=\alpha$ are model dependent coefficients. In addition, $\boldsymbol{H}$ is a 5 -diagonal, symmetric, Toeplitz matrix with elements

$$
h_{i j}= \begin{cases}\frac{1}{4}+2 \alpha^{2} & \text { if } i=j \\ -\alpha & \text { if }|i-j|=1 \\ \alpha^{2} & \text { if }|i-j|=2 \\ 0 & \text { otherwise. }\end{cases}
$$

Finally, $\boldsymbol{Q}_{i} ; i=1,2$, are matrices of the form given in (6.6) with $q_{0}=1$. The parameters $q_{1}=\alpha_{i}$ are model dependent coefficients.

Let the original PSM $\boldsymbol{A}$ be denoted here as $\boldsymbol{A}_{n}$. Once again, the subscript " $n$ " stands for noncausal. On the other hand, the operator $L_{n}$ is given by (6.5) and the image is represented by the vector $f_{n}=f$. In this case, the restored image is obtained as the solution of the following set of linear equations:

$$
\boldsymbol{A}_{R} f_{n}=\boldsymbol{A}_{n}^{T} \boldsymbol{g}
$$

where

$$
\boldsymbol{A}_{R}=\boldsymbol{A}_{n}^{T} \boldsymbol{A}_{n}+\alpha \sum_{i=1}^{L} \sum_{j=1}^{L} \boldsymbol{P}_{i}^{T} \boldsymbol{P}_{j} \otimes \boldsymbol{Q}_{i}^{T} \boldsymbol{Q}_{j} .
$$

The resulting image can be directly obtained by converting the vector $f_{n}$ into a matrix form.

\section{B. Regularized Solutions Based on Semicausal SPDE Image Models}

This section deals with the discrete image restoration based on the representation of the image by semicausal SPDE image models. In this case, the formulation of the image restoration problem is slightly different from the
TABLE III

Discrete Approximation of Noncausal SPDE IMAGE Models

\begin{tabular}{c|c|c}
\hline \hline SPDE & $\begin{array}{l}\text { Partial Differential } \\
\text { Operator } \\
\text { Image Model }\end{array}$ & $\begin{array}{c}\text { Discrete Approximation } \\
L\left(D_{x}, D_{y}\right)\end{array}$ \\
\hline Noncausal 1 (NC1) & $D_{x}^{2}+D_{y}^{2}$ & $\mathbf{L} \otimes \mathbf{Q}+\mathbf{Q} \otimes \mathbf{I}$ \\
\hline Noncausal 2 (NC2) & $\left(D_{x}^{2}+D_{y}^{2}\right)^{2}$ & $\mathbf{I} \otimes \mathbf{H}+\mathbf{H} \otimes \mathbf{I}+2 \mathbf{Q} \otimes \mathbf{Q}$ \\
\hline Noncausal 3 (NC3) & $\left(D_{x}^{2}+\alpha_{1}\right)\left(D_{y}^{2}+\alpha_{2}\right)$ & $\mathbf{Q}_{1} \otimes \mathbf{Q}_{2}$ \\
\hline
\end{tabular}

conventional one, used in the case where the image is represented by noncausal SPDE image models. The new formulation of the problem is mainly dictated by the form of the discrete approximation of the semicausal SPDE image models. The discrete approximation corresponding to semicausal SPDE image models is examined here.

Let $f_{j} ; j=1,2, \cdots, N$ be the $N \times 1$ vectors corresponding to the $N$ columns of the image matrix

$$
F_{n}=\left[f_{1} f_{2} \cdots f_{N}\right] \text {. }
$$

According to [45], the discrete approximation of any semicausal SPDE image model can be expressed by the following set of matrix-vector equations:

$$
\boldsymbol{P}_{1} \boldsymbol{f}_{j}-\boldsymbol{P}_{2} \boldsymbol{f}_{j-1}=\varepsilon_{j} ; \quad j=2,3, \cdots, N
$$

where $\varepsilon_{j} ; j=2,3, \cdots, N$ are $N \times 1$ vectors consisting of zero-mean white noise elements and $\boldsymbol{P}_{1}$ is the $N \times N$ symmetric, tridiagonal, Toeplitz matrix defined by (6.6). The difference between the discrete approximation of the two semicausal SPDE image models proposed in [45] lies on the form of the matrix $\boldsymbol{P}_{2}$. In the case of the nonseparable semicausal SPDE inage model, known as semicausal 1 (SC1),

$$
\boldsymbol{P}_{2}=\gamma \boldsymbol{I}
$$

where $\gamma$ is a model dependent coefficient and $I$ is the $N \times N$ identity matrix. In the case of the separable semicausal SPDE image model, known as semicausal 2 (SC2),

$$
\boldsymbol{P}_{2}=\boldsymbol{P}_{1} \text {. }
$$

Given the above discrete approximation of the semicausal SPDE image models, one possibility is to develop a restoring algorithm estimating each pair of the image columns separately. Using such an approach, the correlation existing in one of the two directions, in fact the horizontal one, is lost. Therefore, even if the algorithm to be developed is optimum in some sense, the entire procedure will be suboptimum. The objective here, therefore, is to develop a restoring algorithm working on the whole image, instead of covering the image estimating the adjacent image columns in successive steps. Therefore, an alternative form of the discrete approximation of semicausal SPDE image models is required; this is now derived. 
Combining the $N-1$ equations given by (6.11), the discrete approximation of any semicausal SPDE image model can be equivalently written in the following form:

$$
\boldsymbol{L}_{s} \boldsymbol{f}_{s}=\boldsymbol{\varepsilon}_{\mathrm{s}} \text {. }
$$

In (6.14), the image is represented by a new $N^{2} \times 1$ vector defined as follows:

$$
\boldsymbol{f}_{s}^{T}=\left[\begin{array}{llll}
\boldsymbol{f}_{1}^{T} & \boldsymbol{f}_{2}^{T} & \cdots & \boldsymbol{f}_{N}^{T}
\end{array}\right]
$$

where $f_{j} ; j=1,2, \cdots, N$ are the image columns. The white noise process associated with any SPDE image model representation participates in $(6.14)$ by means of the following vector:

$$
\boldsymbol{\varepsilon}_{s}^{T}=\left[\begin{array}{lllll}
\mathbf{0}^{T} & \boldsymbol{\varepsilon}_{2}^{T} & \boldsymbol{\varepsilon}_{3}^{T} & \cdots & \boldsymbol{\varepsilon}_{N}^{T}
\end{array}\right]
$$

where $\varepsilon_{j} ; j=2,3, \cdots, N$ are vectors consisting of white noise elements and $\mathbf{0}$ is the $N \times 1$ null vector. Finally, $\boldsymbol{L}_{s}$ is the $N^{2} \times N^{2}$ matrix which carries the identity of the specific SPDE image model, defined as follows:

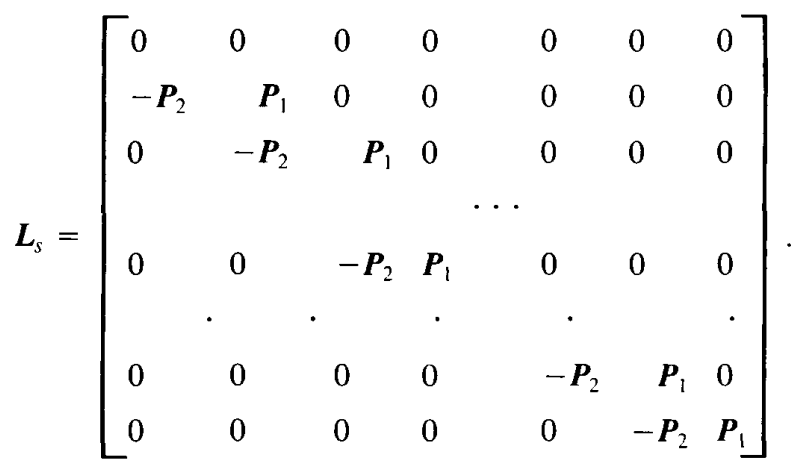

$\boldsymbol{L}_{\mathrm{s}}$ consists of $N \times N$ matrix elements, and $N \times N$ null matrices denoted here by 0 . In fact, the notation used can be significantly simplified because of the special form of the matrix $\boldsymbol{L}_{s}$. Assuming that $\boldsymbol{I}_{N-1}$ is the $(N-1) \times(N$ - 1) identity matrix, define the following $N \times N$ matrices:

$$
\boldsymbol{I}_{1}=\left[\begin{array}{c:c}
0 & \mathbf{0}^{T} \\
\hdashline \mathbf{0} & \boldsymbol{I}_{N-1}
\end{array}\right]
$$

and

$$
\boldsymbol{I}_{2}=\left[\begin{array}{c:c}
\mathbf{0}^{T} & 0 \\
\hdashline \boldsymbol{I}_{N-1} & \mathbf{0}
\end{array}\right]
$$

where $\mathbf{0}$ is the $(N-1) \times 1$ null vector. Taking these definitions into account, it is obvious that $\boldsymbol{L}_{s}$ can be written more elegantly, using the Kronecker product notation, as follows:

$$
L_{s}=I_{1} \otimes P_{1}-I_{2} \otimes P_{2} .
$$

In conclusion, the discrete approximation of the semicausal SPDE image models proposed in [45] is presented
TABLE IV

Discrete Approximation of Semicausal SPDE Image Models

\begin{tabular}{c|c|c}
\hline SPDE & $\begin{array}{l}\text { Partial Differential } \\
\text { Operator }\end{array}$ & Discrete Approximation \\
Image Model & $L\left(D_{x}, D_{y}\right)$ & $\mathbf{L}_{s}$ \\
\hline Semicausal 1 (SC1) & $D_{y}-D_{x}^{2}+\alpha$ & $\mathbf{I}_{1} \otimes \mathbf{Q}-\gamma \mathbf{I}_{2} \otimes \mathbf{I}$ \\
\hline Semicausal 2 (SC2) & $\left(D_{x}^{2}+\alpha_{1}\right)\left(D_{y}+\alpha_{2}\right)$ & $\left(\mathbf{I}_{1}-\mathbf{I}_{2}\right) \otimes \mathbf{Q}$ \\
\hline
\end{tabular}

in Table IV. The importance of this equivalent discrete representation is that the new operator $\boldsymbol{L}_{s}$ operates on the entire image. On the other hand, the discrete image representation model given by $(6.14)$ is of a similar mathematical form with the one corresponding to noncausal SPDE image representation. The only difference is that the image is now represented by the new image vector $f_{s}$, formed from the image matrix as indicated by (6.15).

The general formulation of the image restoration problem is based on the assumption that the image is represented by the same vector in both the discrete approximation of the stabilizing functional as well as the model describing the image formation process. Therefore, the model (1.3) describing the image formation process should be appropriately modified in such a way that the image be again represented in the model by the new vector $f_{s}$. The image vector $f_{n}$, conventionally used to represent an image, consists of $N$ subvectors which correspond to the ordered rows of the image matrix. On the other hand, the image vector $f_{s}$ consists of subvectors which correspond to the columns of the image matrix. Given the form of the new image vector $f_{s}$, the modification of the model describing the image formation process amounts to the appropriate modification of the PSM associated with the image formation model. This modification is described by the following proposition.

Proposition 2: Let $f_{n}$ be the conventional image vector and $f_{s}$ the vector defined by (6.15). Also, let $A_{n}$ be the $N^{2}$ $\times N^{2}$ matrix and $a_{i} ; i=1,2, \cdots, N^{2}$ its columns. Now consider the matrix

$$
\boldsymbol{A}_{s}=\left[\begin{array}{llll}
\boldsymbol{a}_{1}^{\prime} & \boldsymbol{a}_{2}^{\prime} & \cdots & \boldsymbol{a}_{N^{2}}^{\prime}
\end{array}\right]
$$

whose column vectors $\boldsymbol{a}_{i}^{\prime} ; i=1, \cdots, N^{2}$ are related with the column vectors of the original PSM $\boldsymbol{A}_{n}$ as follows:

$$
a_{N(i-1)+j}^{\prime}=a_{N(j-1)+i} \forall 1 \leq i, j \leq N .
$$

Then

$$
\boldsymbol{A}_{s} f_{s}=A_{n} f_{n} .
$$

Proof: The proof of this proposition is given in Appendix E.

The image formation process can be equivalently described by the following discrete model:

$$
\boldsymbol{A}_{s} \boldsymbol{f}_{s}+\boldsymbol{n}=\boldsymbol{g}
$$


where $A_{s}$ is the new PSM, while $f_{s}$ is the new image vector introduced by (6.15). On the other hand, the operator $L_{s}$ is given by $(6.20)$. In this case, the restored image is obtained as the solution of the following set of linear equations:

$$
A_{R} f_{s}=A_{s}^{T} g
$$

where

$$
\boldsymbol{A}_{R}=\boldsymbol{A}_{s}^{T} \boldsymbol{A}_{s}+\alpha \sum_{i=1}^{2} \sum_{j=1}^{2} \mu_{i j} \boldsymbol{I}_{i}^{T} \boldsymbol{I}_{j} \otimes \boldsymbol{P}_{i}^{T} \boldsymbol{P}_{j}
$$

and

$$
\mu_{i j}=\left\{\begin{aligned}
1 & \text { for } i=j \\
-1 & \text { for } i \neq j .
\end{aligned}\right.
$$

The restored image is obtained in this case by converting the image vector $f_{s}$ into a matrix form on the basis of its definition.

\section{Discrete Image Restoration Algorithms}

According to the discrete formulation of the image restoration problem, the restored image is the solution of the following set of linear equations:

$$
A_{R} x=A^{T} g
$$

Serious computational problems arise, in practice, caused by the dimensionality of the resulting set of linear equations. In fact, $A_{R}$ is an $N^{2} \times N^{2}$ matrix while $N=256$ or 512 is a common situation in many of the existing image display systems. Therefore, the direct solution of (6.28), either by evaluating the inverse of $\boldsymbol{A}_{R}$ or by applying any direct method, is impossible for small or medium size computer systems, while impractical, if not impossible, even for large size computer systems. The specific problem of solving a set of linear equations is equivalent with the minimization of a certain quadratic form. A suitable class of iterative methods capable of performing this minimization is the class of gradient methods [47]. The tradeoff existing in choosing iterative algorithms is between the computing requirements, both arithmetic operations per iteration and storage space required, and the rate of convergence. The conjugate gradient algorithm is certainly the most demanding among the existing gradient algorithms, in terms of the computing requirements. However, its rate of convergence is satisfactory in the sense that it converges after a certain number of iterations. In addition, the conjugate gradient algorithm provides very fast convergence in comparison to other gradient algorithms.

The implementation of the conjugate gradient algorithm appears to be rather straightforward [47]. However, the huge size of the matrix $\boldsymbol{A}_{R}$ which needs to be stored makes the applicability of the algorithm in its present form doubtful. The assumption that the image formation process can be described by a separable PSM is particularly important with regard to the implementation of the algorithm. Fortunately, many kinds of distortion appearing in practical situations can be described by a separable PSM as, for instance, linear motion and atmospheric turbulence blur [1], [2]. It is shown in the sequel that assuming a separable PSM, and regardless of the specific SPDE image representation, the original algorithm presented above can be converted into a matrix form.

Consider now the image restoration algorithms based on noncausal SPDE image models, under the assumption that the PSM $\boldsymbol{A}_{n}$ is separable, that is

$$
A_{n}=C \otimes D
$$

where $C$ and $D$ are $N \times N$ matrices. In such a case, (6.9) becomes

$$
\boldsymbol{A}_{R}=\boldsymbol{C}^{T} \boldsymbol{C} \otimes \boldsymbol{D}^{T} \boldsymbol{D}+\alpha \sum_{i=1}^{L} \sum_{j=1}^{L} \boldsymbol{P}_{i}^{T} \boldsymbol{P}_{j} \otimes \boldsymbol{Q}_{i}^{T} \boldsymbol{Q}_{j}
$$

Since $\boldsymbol{A}_{R}$ is a summation of separable matrices, the algorithm can be converted into a matrix form. This algorithm is presented in Table $\mathrm{V}$. The only equations which are affected by the image model used are the equations for the evaluation of $\boldsymbol{R}_{0}$ in the initialization stage of the algorithm, and $\boldsymbol{W}_{k}$ at every iteration.

In the case of image restoration algorithms based on semicausal SPDE image models, the image formation process is described by $(6.24) . f_{s}$ is the new image vector given by (6.15), while $\boldsymbol{A}_{s}$ is related with the original PSM $\boldsymbol{A}_{n}$ as described in Proposition 2. Under the assumption that $\boldsymbol{A}_{n}$ is separable, the form of $\boldsymbol{A}_{s}$ is given by the following proposition.

Proposition 3: Consider that the original PSM $\boldsymbol{A}_{n}$ is separable, given by (6.29). Then, $\boldsymbol{A}_{s}$, defined in Proposition 2 , can be equivalently written as

$$
\boldsymbol{A}_{s}=\left[\begin{array}{c}
\boldsymbol{D} \otimes c_{1}^{T} \\
\boldsymbol{D} \otimes c_{2}^{T} \\
\vdots \\
\boldsymbol{D} \otimes c_{N}^{T}
\end{array}\right]
$$

where $c_{i}^{T} ; i=1, \cdots, N$ are the rows of $C$.

Proof: The proof of this proposition is given in Appendix E.

Taking into account that $\boldsymbol{A}_{s}$ is given by (6.31), it is rather straightforward to show that

$$
\boldsymbol{A}_{s}^{T} \boldsymbol{A}_{s}=\boldsymbol{D}^{T} \boldsymbol{D} \otimes \boldsymbol{C}^{T} \boldsymbol{C} .
$$

Combining (6.26) and (6.32)

$$
\boldsymbol{A}_{R}=\boldsymbol{D}^{T} \boldsymbol{D} \otimes \boldsymbol{C}^{T} \boldsymbol{C}+\alpha \sum_{i=1}^{2} \sum_{j=1}^{2} \mu_{i j} \boldsymbol{I}_{i}^{T} \boldsymbol{I}_{j} \otimes \boldsymbol{P}_{i}^{T} \boldsymbol{P}_{j}
$$

Finally, given that the original PSM is separable, $\boldsymbol{A}_{R}$ is once again a summation of separable matrices. The final step is the expression of the vector $\boldsymbol{A}_{s}^{T} \boldsymbol{g}$ in a matrix form, given by the following proposition. 
TABLE V

Image Restoration Algorithm Based on Noncalsal SPDE Image MODELS

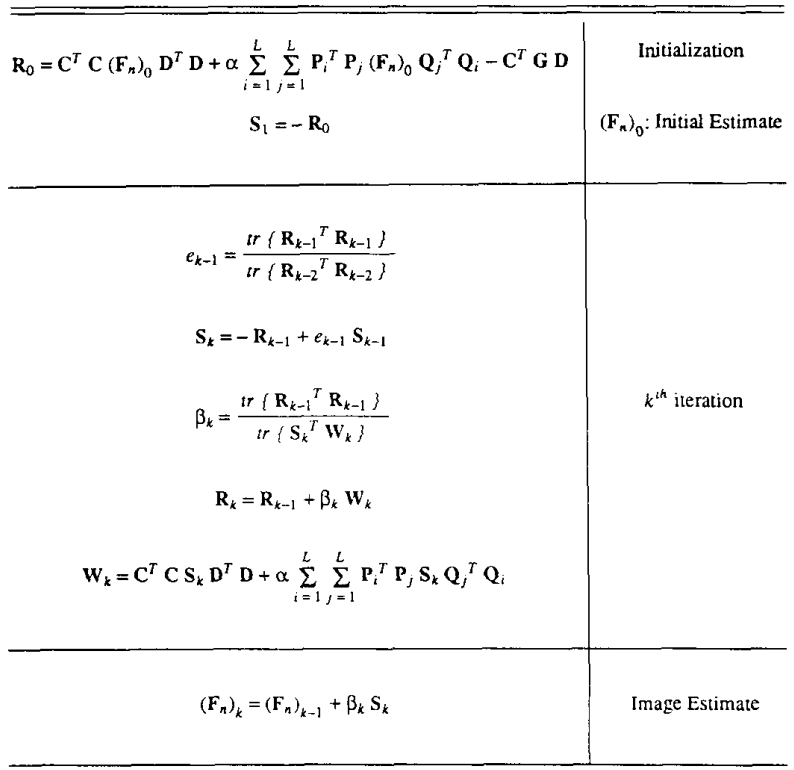

Proposition 4: Consider the vector $\boldsymbol{g}_{R}$, given in terms of $A_{s}$ and the vector $g$ as follows:

$$
\boldsymbol{g}_{R}=\boldsymbol{A}_{s}^{T} \boldsymbol{g} .
$$

Let $\boldsymbol{g}\left(\boldsymbol{g}_{R}\right)$ be formed by lexicographically ordering the elements of the $N \times N$ matrix $\boldsymbol{G}\left(\boldsymbol{G}_{R}\right)$. Then

$$
\boldsymbol{G}_{R}=\boldsymbol{D}^{T} \boldsymbol{G}^{T} \boldsymbol{C} \text {. }
$$

Proof: The proof of this proposition is given in Appendix E.

The restoring algorithm, originally given in a matrixvector form, can be equivalently implemented in a matrix form. This algorithm is presented in Table VI. The only equations which are affected by the image model used are again the equations for the evaluation of $\boldsymbol{R}_{0}$ in the initialization stage of the algorithm, and $\boldsymbol{W}_{k}$ at every iteration.

\section{Regularization and Optimal Wiener ESTIMATION}

The optimum Wiener estimate in image restoration is derived on the basis of the assumption that both the object and the noise belong to homogeneous random fields, and the noise is uncorrelated with the original object [1], [2]. Another implicit assumption considered is that the second-order statistics of the object and the noise are known. However, the a priori knowledge required for the evaluation of the optimum estimate in the Wiener sense is very rarely available [26]. In practical situations, this problem is usually overcome using certain covariance models to approximate the second-order statistics of the object and the noise.

In continuous image restoration, the Wiener filter can be obtained using the additional assumption that the image formation process is described by a space-invariant
TABLE VI

Image Restoration Algorithm Baseid on Semicausal SPDE image

\begin{tabular}{|c|c|}
\hline $\begin{array}{c}\mathbf{R}_{0}=\mathrm{D}^{T} \mathrm{D}\left(\mathbf{F}_{s}\right)_{0} \mathrm{C}^{T} \mathrm{C}+\alpha \sum_{i=1}^{2} \sum_{j=1}^{2} \mu_{i j} \mathbf{I}_{i}^{T} \mathbf{I}_{j}\left(\mathbf{F}_{s}\right)_{0} \mathbf{P}_{j}^{T} \mathbf{P}_{i}-\mathbf{D}^{T} \mathbf{G}^{T} \mathrm{C} \\
\mathbf{S}_{1}=-\mathbf{R}_{0}\end{array}$ & $\begin{array}{l}\text { Initialization } \\
\left(F_{s}\right)_{0}: \text { Initial Estimate }\end{array}$ \\
\hline$e_{k-1}=\frac{\operatorname{tr}\left(\mathbf{R}_{k-1}{ }^{T} \mathbf{R}_{k-1}\right\}}{\operatorname{tr}\left\{\mathbf{R}_{k-2}{ }^{T} \mathbf{R}_{k-2}\right\}}$ & \\
\hline $\mathbf{S}_{\boldsymbol{k}}=-\mathbf{R}_{k-1}+e_{k-1} \mathbf{S}_{k-1}$ & \\
\hline$\beta_{k}=\frac{\operatorname{tr}\left\{\mathbf{R}_{k-1}{ }^{T} \mathbf{R}_{k-1}\right\}}{\operatorname{tr}\left\{\mathbf{S}_{k}^{T} \mathbf{W}_{k}\right\}}$ & $k^{\text {ih }}$ iteration \\
\hline $\begin{array}{c}\mathbf{R}_{k}=\mathbf{R}_{k-1}+\beta_{k} \mathbf{W}_{k} \\
\mathbf{W}_{k}=\mathbf{D}^{T} \mathbf{D} \mathbf{S}_{k} \mathbf{C}^{T} \mathbf{C}+\alpha \sum_{i=1}^{2} \sum_{j=1}^{2} \mu_{i j} \mathbf{I}_{i}^{T} \mathbf{I}_{j} \mathbf{S}_{k} \mathbf{P}_{j}^{T} \mathbf{P}_{i}\end{array}$ & \\
\hline$\left(\mathbf{F}_{s}\right)_{k}=\left(\mathbf{F}_{s}\right)_{k-1}+\beta_{k} S_{k}$ & Image Estimate \\
\hline
\end{tabular}
MODELS

model as follows [1]:

$$
H_{W}\left(\omega_{1}, \omega_{2}\right)=\frac{H^{*}\left(\omega_{1}, \omega_{2}\right)}{\left|H\left(\omega_{1}, \omega_{2}\right)\right|^{2}+\frac{S_{n n}\left(\omega_{1}, \omega_{2}\right)}{S_{f f}\left(\omega_{1}, \omega_{2}\right)}} .
$$

Where $S_{f f}(\cdot)$ and $S_{n n}(\cdot)$ are the spectral densities of the object and noise, respectively. Let $f(x, y)$ be a 2-D stochastic process and $\epsilon(x, y)$ be a 2-D zero-mean white noise process with variance $\sigma_{\epsilon}^{2}$. Consider that the stochastic processes $f(\cdot)$ and $\epsilon(\cdot)$ are related by the following SPDE:

$$
\begin{aligned}
& L\left(D_{x}, D_{y}\right) f(x, y) \\
& \quad=\sum_{p=0}^{K} \sum_{q=0}^{L} \lambda_{p, q} \frac{\partial^{p+q} f(x, y)}{\partial x^{p} \partial y^{q}}=\epsilon(x, y) .
\end{aligned}
$$

Assuming that $f(\cdot)$ corresponds to an image, (7.2) is its SPDE model representation. The SPDE (7.2) defines a 2-D linear system with input $\epsilon(\cdot)$, output $f(\cdot)$, and system function

$$
T\left(s_{1}, s_{2}\right)=\frac{1}{L\left(s_{1}, s_{2}\right)}
$$

where

$$
L\left(s_{1}, s_{2}\right)=\sum_{p=0}^{K} \sum_{q=0}^{L} \lambda_{p, q} s_{1}^{p} s_{2}^{q}
$$

Therefore, an image represented by $f(\cdot)$ can be considered as the output of the linear system defined above, driven by a white noise process $\epsilon(\cdot)$. From the fundamental relation concerning linear systems with input and output stochastic processes, the spectral densities of the input and output of the linear system defined above are 
related as follows:

$$
\begin{aligned}
S_{f f}\left(\omega_{1}, \omega_{2}\right) & =\frac{1}{\left|L\left(j \omega_{1}, j \omega_{2}\right)\right|^{2}} S_{\epsilon \epsilon}\left(\omega_{1}, \omega_{2}\right) \\
& =\frac{\sigma_{\epsilon}^{2}}{\left|L\left(j \omega_{1}, j \omega_{2}\right)\right|^{2}} .
\end{aligned}
$$

Taking into account (7.5), and also assuming that the noise $n(\cdot)$ associated with the image formation is a zeromean white process with variance $\sigma_{n}^{2}$

$$
\frac{S_{n n}\left(\omega_{1}, \omega_{2}\right)}{S_{f f}\left(\omega_{1}, \omega_{2}\right)}=\frac{\sigma_{n}^{2}}{S_{f f}\left(\omega_{1}, \omega_{2}\right)}=\alpha\left|L\left(j \omega_{1}, j \omega_{2}\right)\right|^{2}
$$

where

$$
\alpha=\frac{\sigma_{n}^{2}}{\sigma_{\epsilon}^{2}} .
$$

Consider that an image can be represented by the SPDE model (7.2). The relationship determined by (7.6) provides a link between the class of RF's defined by $(5.5)$ and Wiener filtering. The RF of the form (5.5) with regularizing polynomial given by (5.7) is the optimum Wiener filter for this image. On the other hand, (7.6) determines the relationship between the class of GRF's defined by (5.17) and the power spectrum equalization filter, derived on the basis of the assumption that the power spectrum of the image estimate is equal to the power spectrum of the original image [1]. The GRF of the form (5.17) with regularizing polynomial given by $(5.7)$ is the optimum power spectrum equalization filter for this image.

The relationship between Wiener estimation and the regularized solutions resulting from the image representation by SPDE image models is now investigated for the discrete image restoration case. The optimum Wiener estimate for the original object is given in this case as follows [1], [2]:

$$
\hat{f}=\boldsymbol{K}_{f f} \boldsymbol{A}^{*}\left(\boldsymbol{A} \boldsymbol{K}_{f f} \boldsymbol{A}^{*}+\boldsymbol{K}_{n n}\right)^{-1} \boldsymbol{g}
$$

In (7.8), $\boldsymbol{K}_{f f}$ and $\boldsymbol{K}_{n n}$ are the covariance matrices for the object and the noise, respectively. Assuming that the noise $n$ is white with variance $\sigma_{n}^{2}$

$$
\boldsymbol{K}_{n n}=\sigma_{n}^{2} \boldsymbol{I}
$$

Combining (7.8) and (7.9)

$$
\begin{aligned}
\hat{\boldsymbol{f}} & =\boldsymbol{K}_{f f} \boldsymbol{A}^{*}\left(\boldsymbol{A} \boldsymbol{K}_{f f} \boldsymbol{A}^{*}+\sigma_{n}^{2} \boldsymbol{I}\right)^{-1} \boldsymbol{g} \\
& =\left(\boldsymbol{A}^{*} \boldsymbol{A}+\sigma_{n}^{2} \boldsymbol{K}_{f f}^{-1}\right)^{-1} \boldsymbol{A}^{*} \boldsymbol{g} .
\end{aligned}
$$

Assume that the original object can be represented by the discrete approximation of an SPDE image model, that is

$$
L f=\varepsilon
$$

where $\boldsymbol{L}$ is a model dependent matrix operator and $\boldsymbol{\varepsilon}$ is a white noise process with variance $\sigma_{f}^{2}$. From (7.11)

$$
\begin{aligned}
\boldsymbol{K}_{\epsilon t} & =E\left\{\boldsymbol{\varepsilon} \boldsymbol{\varepsilon}^{*}\right\}=E\left\{\boldsymbol{L} f f^{*} \boldsymbol{L}^{*}\right\} \\
& =\boldsymbol{L} E\left\{f f^{*}\right\} \boldsymbol{L}^{*}=\boldsymbol{L} \boldsymbol{K}_{f f} \boldsymbol{L}^{*} .
\end{aligned}
$$

According to the assumption regarding the noise process $\varepsilon$

$$
\boldsymbol{K}_{\mathrm{\epsilon \epsilon}}=\sigma_{\epsilon}^{2} \boldsymbol{I}
$$

Combining (7.12) and (7.13)

$$
\sigma_{\epsilon}^{2} \boldsymbol{I}=\boldsymbol{L} \boldsymbol{K}_{f f} \boldsymbol{L}^{*}
$$

or

$$
\boldsymbol{K}_{f f}=\sigma_{\epsilon}^{2}\left(\boldsymbol{L}^{*} \boldsymbol{L}\right)^{-1}
$$

Taking into account (7.10) and (7.15), it can easily be shown that

$$
\hat{f}=\left(A^{*} \boldsymbol{A}+\alpha L^{*} \boldsymbol{L}\right)^{-1} \boldsymbol{A}^{*} \boldsymbol{g}
$$

where $L$ is related to the particular SPDE image model representation considered by (7.11), and $\alpha$ is again defined by (7.7).

Consider that an image can be represented by the model (7.11). The regularized solution given by (7.16) is the optimum Wiener estimate for this image. In conclusion, the optimality of this class of regularized solutions is related to the image representation by an SPDE model. Therefore, the problem of determining the specific SPDE model which provides the best image representation is of particular importance. In other words, the search for satisfactory regularized solutions is closely related to the search for the specific SPDE image models which provide satisfactory image representation.

\section{Experimental Results}

The Aphrodite of Milos, shown in Fig. 1, was used as a test image in the set of experiments based on the regularizing filters proposed in Section V. The distorted image is shown in Fig. 2. The distortion used is the one corresponding to horizontal linear motion blur, as modeled in [1]. In addition to blur, the image was contaminated by additive Gaussian noise. The SNR determined with respect to the undegraded image is $25 \mathrm{~dB}$. The restored images reported here were obtained by filtering the distorted image using the GRF whose transfer function is given by (5.17). Figs. 3 and 4 show the restored images which correspond to the regularizing polynomials based on the $\mathrm{NCl}$ and $\mathrm{SCl}$ image models, respectively. Several experiments indicated that the use of different regularizing polynomials causes a significant variation to the quality of the resulting image estimates. The regularizing polynomials corresponding to SCl (semicausal 1) and NCl (Poisson) image models result in very good and visually pleasing restored images. The regularizing polynomial corresponding to NC2 (biharmonic) image model results in a restored image whose visual quality is slightly inferior. Finally, several experiments showed that the regularizing polynomials corresponding to $\mathrm{C} 1, \mathrm{SC} 2$, and $\mathrm{NC} 3$ image models result in restored images of relatively poor visual quality. The filters proposed are sensitive to the level of the noise in the distorted image. It has been estimated that these filters result in visually satisfactory images only for SNR greater than $20 \mathrm{~dB}$. In terms of the computational 


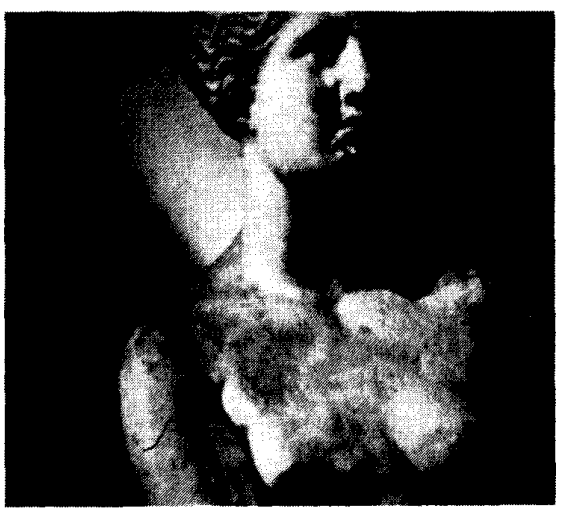

Fig. 1. Original image (the statue of Aphrodite of Milos).

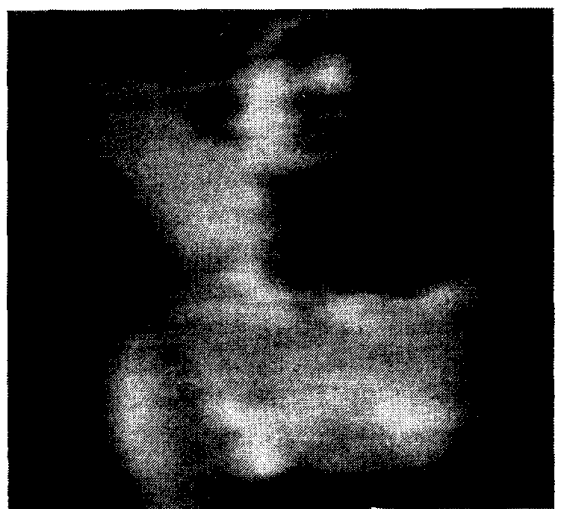

Fig. 2. Distorted image. The distortion consists of horizontal linear motion blur and additive Gaussian noise.

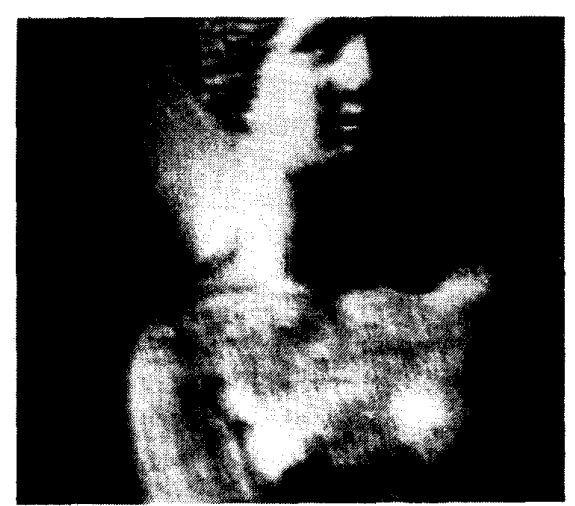

Fig. 3. Restored image by the GRF defined by (5.17) which corresponds to the $\mathrm{NCl}$ image model $\left(\alpha=1 \times 10^{5}\right)$.

requirements, these filters are very fast due to the existence of very fast algorithms performing 2-D discrete Fourier transform. For example, the time required for the restoration of a $256 \times 256$ image in the Vax $11 / 780$ computer system was approximately 3 minutes CPU time. The time required for restoration, in addition to the simple realization of such filters, make them ideal for use in small and medium size computer systems.

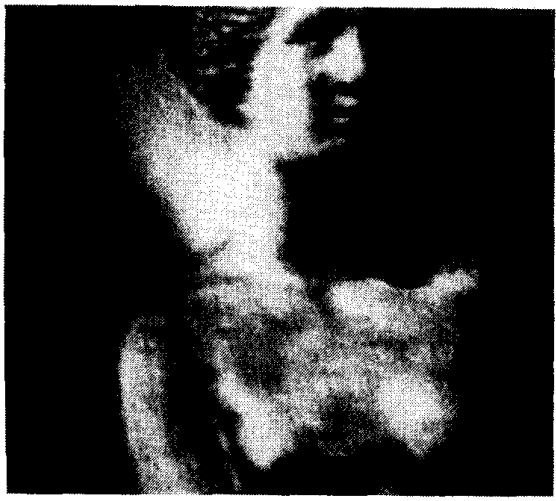

Fig. 4. Restored image by the GRF defined by (5.17) which corresponds to the SC1 image model $\left(\alpha=1 \times 10^{-5}\right)$

The GRF's corresponding to $\gamma=\frac{1}{2}$ provide the best results among all the regularizing filters proposed in Section V. A remarkable case is the experimental performance evaluation of the class of regularizing filters defined by (5.5). Despite the optimal derivation of this class of filters, their performance is not satisfactory. In fact, the restored image obtained using this class of filters was not found to be pleasing to the human eye, although the blur and noise are reduced substantially. This is not surprising, taking into account the optimality of this class of filters in the Wiener sense presented in Section VIl. It has been pointed out very early that the performance of Wiener filtering, when performed in the frequency domain, suffers in terms of the resulting visual quality [1].

The Aphrodite of Milos, shown in Fig. 5, was again the test image in the set of experiments based on the discrete algorithms presented in Section VI. The distorted image is shown in Fig. 6. The distortion appearing is a horizontal linear motion blur, as modeled in [7]. The image was also contaminated by additive Gaussian noise. The experiments indicated that the discrete algorithms based on nonseparable SPDE image models result in better restored images than the ones based on separable models. The superiority of the nonseparable SPDE image models over the separable ones becomes obvious in situations of severe degradation and/or noise. Figs. 7 and 8 show the best restored images, obtained by employing the discrete algorithms corresponding to the $\mathrm{NCl}$ and $\mathrm{SCl}$ image models, respectively.

In these experiments, the iterative algorithms were initialized by using an identically zero matrix as the initial estimate of the restored image. Such an initial estimate is widely used in the application of iterative gradient methods in image processing problems [22]. The conjugate gradient algorithm used in these experiments converges to a satisfactory image estimate after a certain number of iterations. It was experimentally found that the number of 50 iterations satisfies the tradeoff between the visual quality of the restored image and the computer time required. In fact, there exists some improvement of the restored image after 50 iterations, in particular, the enhancement of some fine details of the image. However, the improve- 


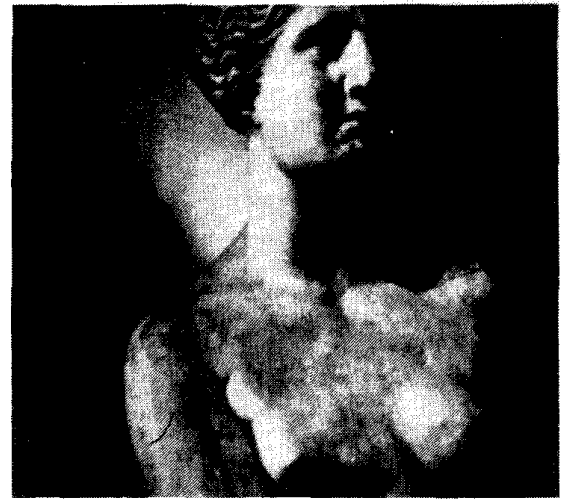

Fig. 5. Original image (the statue of Aphrodite of Milos).

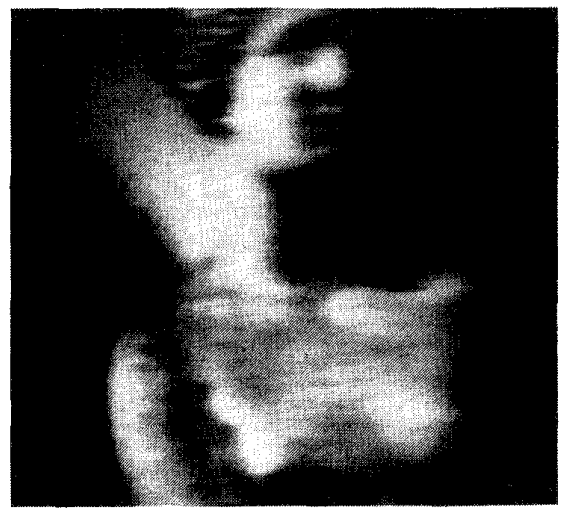

Fig. 6. Distorted image. The distortion consists of horizontal linear motion blur and additive Gaussian noise.

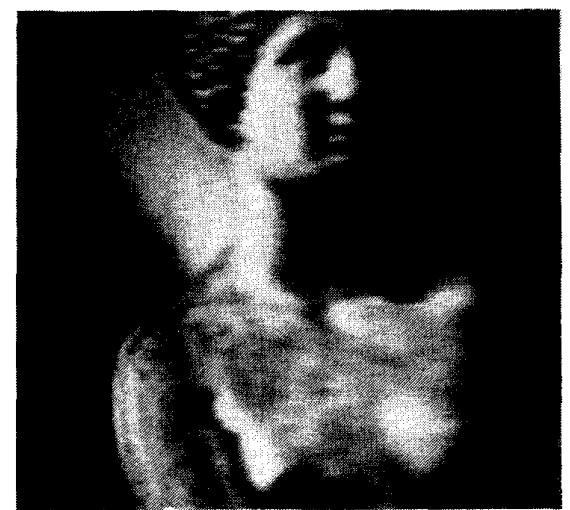

Fig. 7. Restored image by the algorithm which corresponds to the $\mathrm{NCl}$ image model $\left(\alpha=1 \times 10^{-4} ; 50\right.$ iterations $)$.

ment is not as substantial as to be considered worth the computer time required. Another alternative examined during the experimental work was to make the algorithms independent from a predecided number of iterations. In such a case, the algorithm may stop by itself using as a criterion that the error measurement provided by the con-

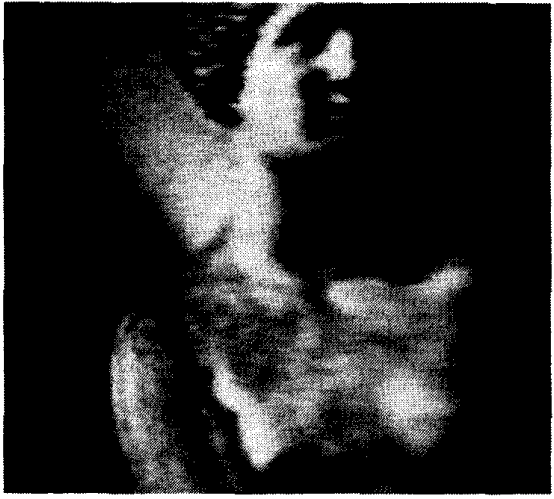

Fig. 8. Restored image by the algorithm which corresponds to the $\mathrm{SCl}$ image model ( $\alpha=1 \times 10^{-4} ; 50$ iterations).

jugate algorithm be smaller than a given threshold. However, the main difficulty arising is the lack of an obvious correspondence between any threshold chosen and the visual quality of the image.

Discrete image restoration is by its nature computationally very demanding, due to the huge amount of data involved in the image formation process. In fact, the implementation of any discrete algorithm for image restoration involves several multiplications of very largesize matrices. On the other hand, the conjugate gradient algorithm, by itself, requires an excessive amount of storage space for its implementation. Fortunately, all matrices involved in the discrete approximation of SPDE image models are sparse matrices possessing a standard form. Therefore, with some additional programming effort, a lot of arithmetic operation can be avoided by ignoring all multiplications including zeros. On the other hand, due to the implementation of the algorithms in a matrix form, the largest matrix to be stored is of size $N \times N$.

According to the experiments, the regularizing parameter $\alpha$ controls the tradeoff between noise reduction and the restoration of the fine details of the image. The value of $\alpha$ is mainly affected from the level of noise in the degraded image. The higher the level of noise in the degraded image is, the larger the parameter $\alpha$ will be. On the other hand, the larger the parameter $\alpha$ is, the higher the degree of smoothness in the restored image will be. Therefore, the choice of $\alpha$ may be based on a subjective quality criterion.

According to the experimental results, any stabilizing functional used amounts to stable image estimates. However, the visual quality of the results is not guaranteed by the use of any stabilizing functional. This supports the argument that regularization theory, although guaranteeing the stability of the image estimates, cannot be used alone to solve the image restoration problem. In other words, this is an experimental verification of the importance of the MAP estimation interpretation and the use of SPDE image models proposed in this paper. Finally, this set of experimental results can also be used for the qual- 
itative evaluation of various SPDE image models in representing images. In fact, the resulting evaluation exhibits full agreement with the one presented in [45]. As follows from the experiments, the SC1 and NC1 image models provide the best image representation. Some additional comments are as follows:

1) Image representation using a causal SPDE image model is not realistic. This is not surprising since, using such a representation, each pixel is correlated with only the "past" pixels in both directions, although every pixel is correlated with all its neighbors.

2) Semicausal and noncausal SPDE image models provide the most realistic image representation.

3) Nonseparable SPDE image models are the most appropriate for image representation. A direct implication of this is that the best stabilizing functionals correspond to nonseparable PDO's as well.

\section{Conclusions}

This paper examined several aspects of regularization theory in image restoration; this was accomplished by extending the applicability of the stabilizing functional approach to $2-\mathrm{D}$ inverse problems. It was shown that the stabilizing functional approach can be the basis for the derivation of a large variety of regularized solutions in image restoration, depending on the form of the stabilizing functional chosen. A systematic study regarding the choice of stabilizing functionals was also presented, with particular emphasis on a certain class of quadratic functionals. The particular class of quadratic functionals whose minimization amounts to 2-D $L$-generalized splines was finally considered the source of stabilizing functionals. This particular class of quadratic functionals was also interpreted as a link between the family of constrained least squares and the stabilizing functional approach. The choice of a particular quadratic stabilizing functional was related to the a priori information regarding the expected regularized solution. More specifically, the MAP estimation interpretation of the proposed formulation indicated that there exists a one-to-one correspondence between any quadratic functional used and a certain SPDE image model representation. This MAP estimation formulation also provides the basis for a stochastic interpretation of the entire family of constrained least squares approaches. The optimality of the resulting regularized solutions in the Wiener sense was finally discussed. The importance of the work presented in this paper is that several mathematical concepts and statistical approaches, which appeared independently toward the solution of the image restoration problem, converged to the derivation of a variety of analytically optimum regularized solutions that can be related to the stochastic nature of the image restoration problem.

\section{Appendix A}

Proof of Theorem 1: The quadratic functional defined by $(2.10)$ is the particular element of the class of functionals defined by $(2.4)$ for which

$$
F=\sum_{k, m=0}^{K} \sum_{l, n=0}^{L} f_{x^{k}, y^{\prime}} q_{k, l ; m, n} f_{x^{m}, y^{n}}
$$

The second variation of any functional of the form (2.4) is given by [29]

$\delta^{2} \Omega[h]=\frac{1}{2} \iint\left(\sum_{i, k=0}^{K} \sum_{j, i=0}^{L} \frac{\partial^{2} F}{\partial f_{x^{i} y^{j}} \partial f_{x^{k} y^{\prime}}} h_{x^{i} y^{j}} h_{x^{k} y^{\prime}}\right) d x d y$.

In the particular case that $\Omega[f]=Q[f], F$ is given by (A1). Therefore,

$$
\frac{\partial^{2} F}{\partial f_{x^{i} y^{j}} \partial f_{x^{k} y^{\prime}}}=2 q_{i, j ; k, l}
$$

Hence

$$
\begin{aligned}
\delta^{2} Q[h] & =\iint\left(\sum_{i, k=0}^{K} \sum_{j, l=0}^{L} h_{x^{j} y_{j} j} q_{i, j ; k, l} h_{x^{k} y^{\prime}}\right) d x d y \\
& =Q[h] .
\end{aligned}
$$

Assuming that $q_{k, m, l, n}=q_{l, n: k, m}$, (2.12) can be obtained by combining (2.7) and (A1) as follows:

$$
\begin{aligned}
Q^{\prime}[f]= & \frac{1}{2} \sum_{k=0}^{K} \sum_{l=0}^{L}(-1)^{k+l} \frac{\partial^{k+l}}{\partial x^{k} \partial y^{l}} \\
& \cdot\left\{2 \sum_{m=0}^{K} \sum_{n=0}^{L} q_{k, l: m, n} \frac{\partial^{m+n} f}{\partial x^{m} \partial y^{n}}\right\} \\
= & \sum_{k=0}^{K} \sum_{l=0}^{L}(-1)^{k+l} \sum_{m=0}^{K} \sum_{n=0}^{L} q_{k, l i m, n} D_{x}^{k+m} D_{y}^{l+n} f \\
= & B\left(D_{x}, D_{y}\right) f(x, y)
\end{aligned}
$$

where $B(\cdot)$ is the PDO defined in (2.13).

Assume now that $f \in C_{0}^{(K, L)}[R]$. Since all derivatives of $F(\cdot)$ of order higher than two vanish

$$
\Delta Q[h]=Q[f+h]-Q[f]=\delta Q[h]+\delta^{2} Q[h] .
$$

Since $\delta^{2} Q[h]=Q[h]$

$$
\Delta Q[h]=\delta Q[h]+Q[h] .
$$

For $f=h$

$\Delta Q[h]=Q[2 h]-Q[h]=4 Q[h]-Q[h]=3 Q[h]$.

Taking into account (A5), the first variation of the functional defined by $(2.10)$ is given by

$$
\delta Q[h]=2\left\langle h, Q^{\prime}[f]\right\rangle=2\langle h, B f\rangle .
$$

The combination of (A7), (A8), and (A9) amounts to

$$
Q[h]=\frac{1}{2} \delta Q[h]=\langle h, B f\rangle .
$$

Equation (2.14) can easily be obtained from (A10) by using the substitution $h=f$. This substitution is an allowable one, since it was assumed that $f \in C_{0}^{(K, L)}[R]$. The 
resulting equation (2.14) completes the proof of the theorem.

\section{APPENDIX B}

Assume that $f_{0}$ minimizes the smoothing functional (3.1). Then, for $f=f_{0}$

$$
\begin{aligned}
\delta M_{\alpha}[h, g] & =\delta\|A h-g\|^{2}+\alpha \delta \Omega[h] \\
& =0 \forall h \in C_{0}^{(K . L)}[R] .
\end{aligned}
$$

Consider the following increment:

$$
\begin{aligned}
\Delta\|A h-g\|^{2} & =\|A(f+h)-g\|^{2}-\|A f-g\|^{2} \\
& =2\left\langle h, A^{*}(A f-g)\right\rangle+\|A h\|^{2} .(\mathrm{B} 2)
\end{aligned}
$$

By definition [27], the first variation of $\|A f-g\|^{2}$ is the linear functional with respect to $h$ appearing in (B2). Therefore

$$
\delta\|A h-g\|^{2}=2\left\langle h, A^{*}(A f-g)\right\rangle .
$$

The first variation of $\Omega[f]$ is given by (2.6). Combining (B3) and (2.6), the necessary condition (B1) amounts to

$$
\left\langle h, A^{*} A f_{0}+\alpha \Omega^{\prime}\left[f_{0}\right]-A^{*} g\right\rangle=0 \forall h \in C_{0}^{(K, L)}[R] .
$$

According to the basic lemma of the calculus of variations [27], (B4) amounts to

$$
A^{*} A f_{0}+\alpha \Omega^{\prime}\left[f_{0}\right]=A^{*} g .
$$

Suppose that a nonnegative quadratic functional of the form $(2.10)$ is used as a stabilizing functional. For $\Omega[f]$ $=Q[f]$, the smoothing functional (3.1) becomes

$$
M_{\alpha}[f, g]=\|A f-g\|^{2}+\alpha Q[f] .
$$

Combining (B5) and (2.12), the necessary condition (B5) amounts to

$$
\left(A^{*} A+\alpha B\right) f_{0}=A^{*} g .
$$

The condition (B7) is also sufficient if, for any $f \neq f_{0}$

$$
M_{\alpha}[f, g]-M_{\alpha}\left[f_{0}, g\right] \geq 0 .
$$

Without loss of generality, assume that for some $h \in$ $C^{(K, L)}[R], f=f_{0}+h$. Then

$$
\begin{aligned}
\Delta M_{\alpha}[h, g] & =M_{\alpha}\left[f_{0}+h, g\right]-M_{\alpha}\left[f_{0}, g\right] \\
& =\Delta\|A h-g\|^{2}+\alpha \Delta Q[h] .
\end{aligned}
$$

According to (B2)

$$
\Delta\|A h-g\|^{2}=\delta\|A h-g\|^{2}+\|A h\|^{2} .
$$

Since $Q[\cdot]$ is a quadratic functional, the terms involving derivatives of order higher than two vanish. Therefore

$$
\Delta Q[h]=\delta Q[h]+\delta^{2} Q[h] .
$$

Taking into account (B10) and (B11), (B9) amounts to

$$
\Delta M_{\alpha}[h, g]=\delta M_{\alpha}[h, g]+\|A h\|^{2}+\alpha \delta^{2} Q[h] .
$$

Since $\delta M_{\alpha}[h, g]=0 \forall h \in C_{0}^{(K, L)}[R]$

$$
\Delta M_{\alpha}[h, g]=\|A h\|^{2}+\alpha \delta^{2} Q[h] .
$$

Taking into account (2.11), the nonnegative definition of $Q[\cdot]$ implies that

$$
\delta^{2} Q[h]=Q[h] \geq 0 .
$$

Since $\alpha>0$, the combination of (B13) and (B14) indicates that (B8) is valid. Therefore, (B7) is also a sufficient condition.

\section{Appendix C}

Proof of Proposition 1: Consider the PDE which corresponds to the $\operatorname{PDO} L(\cdot)$, that is

$$
u(x, y)=L\left(D_{x}, D_{y}\right) f(x, y) .
$$

The element $u_{i, j}=u(i h, j h)$ corresponding to the ( $i$, $j$ ) node of the image can be approximated as follows:

$$
u_{i, j}=\sum_{k=0}^{K} \sum_{l=0}^{L} \lambda_{k, l} f_{x^{k} y^{\prime}}(i, j) \forall 1 \leq i, j \leq N .
$$

Taking into account (3.13), (C2) amounts to

$$
u_{i, j}=\sum_{k=0}^{K} \sum_{l=0}^{L} \lambda_{k, l} \xi_{k, l}^{T} f(i, j) \forall 1 \leq i, j \leq N .
$$

Let $\boldsymbol{u}$ be the $N^{2} \times 1$ vector consisting of all $u_{i, j} ; 1 \leq i$, $j \leq N$. Let also $f$ and $f^{\prime}$ be the vectors defined in Section III. Since all elements of $f(i, j) ; 1 \leq i, j \leq N$ belong to either $f$ or $f^{\prime}$, there exists an $N^{2} \times N^{2}$ matrix $\boldsymbol{L}$ such that $(\mathrm{C} 3)$ is equivalent to the following:

$$
\boldsymbol{u}=\boldsymbol{L} f+\boldsymbol{f}^{\prime} .
$$

Neglecting the effect of the boundary elements, (C4) can be written as follows:

$$
\boldsymbol{u}=\boldsymbol{L f}
$$

which is, in fact, the discrete approximation of the PDE (C1).

The discrete approximation of any quadratic functional is given by (3.14). For $q_{k, l: m, n}=\lambda_{k, l} \lambda_{m, n}$, (3.14) amounts to

$$
\begin{aligned}
Q_{L}^{d}[f]= & \sum_{i=1}^{N} \sum_{j=1}^{N} \sum_{k, m=0}^{K} \sum_{l, n=0}^{L} \boldsymbol{f}(i, j)^{T} \\
& \cdot \boldsymbol{\xi}_{k, l} \lambda_{k, l} \lambda_{m, n} \boldsymbol{\xi}_{m, n}^{T} \boldsymbol{f}(i, j) \\
= & \sum_{i=1}^{N} \sum_{j=1}^{N} \sum_{k=0}^{K} \sum_{l=0}^{L} \lambda_{k, l} \boldsymbol{f}(i, j)^{T} \\
& \cdot \boldsymbol{\xi}_{k, l} \sum_{m=0}^{K} \sum_{n=0}^{L} \lambda_{m, n} \xi_{m, n}^{T} \boldsymbol{f}(i, j) .
\end{aligned}
$$

Taking into account (C3), (C6) becomes

$$
Q_{L}^{d}[f]=\sum_{i=1}^{N} \sum_{j=1}^{N} u_{i, j}^{2}=\boldsymbol{u}^{T} \boldsymbol{u}=\boldsymbol{f}^{T} \boldsymbol{L}^{T} \boldsymbol{L} \boldsymbol{f}=\|\boldsymbol{L} \boldsymbol{f}\|^{2}
$$

which proves the proposition. 


\section{APPENDIX D}

Derivation of Regularizing Filters

Taking the Fourier transform of both sides of (5.2)

$$
\begin{gathered}
\bar{H}\left(\omega_{1}, \omega_{2}\right) F\left(\omega_{1}, \omega_{2}\right)+\alpha \mathfrak{F}\left\{B\left(D_{x}, D_{y}\right) f(x, y)\right\} \\
=\bar{G}\left(\omega_{1}, \omega_{2}\right)
\end{gathered}
$$

where $\bar{H}(\cdot)$ and $\bar{G}(\cdot)$ denote the Fourier transform of $\bar{h}(\cdot)$ and $\bar{g}(\cdot)$, respectively. In the derivation which follows, $H(\cdot)$ and $G(\cdot)$ denote the Fourier transform of $h(\cdot)$ and $g(\cdot)$, respectively. By definition

$$
\begin{aligned}
\bar{H}\left(\omega_{1}, \omega_{2}\right) & =\iint \bar{h}(x, y) e^{-j \omega_{1} x} e^{-j \omega_{2} y} d x d y \\
& =\iint h(\xi, \zeta) h^{\prime}\left(\xi, \zeta, \omega_{1}, \omega_{2}\right) d \xi d \zeta
\end{aligned}
$$

where

$$
\begin{aligned}
& h^{\prime}\left(\xi, \zeta, \omega_{1}, \omega_{2}\right) \\
& \quad=\iint h(\xi-x, \zeta-y) e^{-j \omega_{1} x} e^{-j \omega_{2} y} d x d y .
\end{aligned}
$$

Set $\xi-x=\rho, \zeta-y=\phi$. Then

$$
h^{\prime}\left(\xi, \zeta, \omega_{1}, \omega_{2}\right)
$$

$$
\begin{aligned}
& =\iint h(\rho, \phi) e^{-\omega_{1}(\xi-\rho)} e^{-j \omega_{2}(\zeta-\phi)} d \rho d \phi \\
& =e^{-j \omega_{1} \xi} e^{-j \omega_{2} \zeta} H^{*}\left(\omega_{1}, \omega_{2}\right) .
\end{aligned}
$$

Combining (D2) and (D4)

$$
\begin{aligned}
\bar{H}\left(\omega_{1}, \omega_{2}\right)= & H^{*}\left(\omega_{1}, \omega_{2}\right) \iint h(\xi, \zeta) \\
& \cdot e^{-j \omega_{1} \xi} e^{-j \omega_{2} \zeta} d \xi d \zeta=\left|H\left(\omega_{1}, \omega_{2}\right)\right|^{2} .
\end{aligned}
$$

In addition

$$
\begin{aligned}
\bar{G}\left(\omega_{1}, \omega_{2}\right) & =\iint \bar{g}(x, y) e^{-j \omega_{1} x} e^{-j \omega_{2} y} d x d y \\
& =\iint g(\xi, \zeta) h^{\prime}\left(\xi, \zeta, \omega_{1}, \omega_{2}\right) d \xi d \zeta
\end{aligned}
$$

where $h^{\prime}(\cdot)$ is given by (D4). Combining (D4) and (D6)

$$
\begin{aligned}
\bar{G}\left(\omega_{1}, \omega_{2}\right)= & H^{*}\left(\omega_{1}, \omega_{2}\right) \iint g(\xi, \zeta) \\
& \cdot e^{-j \omega_{1} \xi} e^{-j \omega_{2} \zeta} d \xi d \zeta \\
= & H^{*}\left(\omega_{1}, \omega_{2}\right) G\left(\omega_{1}, \omega_{2}\right) .
\end{aligned}
$$

Using the properties of Fourier transform

$$
\mathfrak{F}\left\{B\left(D_{x}, D_{y}\right) f(x, y)\right\}=R\left(\omega_{1}, \omega_{2}\right) F\left(\omega_{1}, \omega_{2}\right)
$$

where

$$
R\left(\omega_{1}, \omega_{2}\right)=B\left(j \omega_{1}, j \omega_{2}\right)
$$

is the regularizing polynomial corresponding to the PDO $B(\cdot)$. Substituting (D5), (D7), and (D8) in (D1)

$$
\begin{gathered}
{\left[\left|H\left(\omega_{1}, \omega_{2}\right)\right|^{2}+\alpha R\left(\omega_{1}, \omega_{2}\right)\right] F\left(\omega_{1}, \omega_{2}\right)} \\
=G\left(\omega_{1}, \omega_{2}\right) H^{*}\left(\omega_{1}, \omega_{2}\right) .
\end{gathered}
$$

Equation (D10) determines the regularizing filter which corresponds to the PDO $B(\cdot)$. The transfer function of this filter is obtained as follows:

$$
\begin{aligned}
H_{R}\left(\omega_{1}, \omega_{2}, \alpha\right) & =\frac{F\left(\omega_{1}, \omega_{2}\right)}{G\left(\omega_{1}, \omega_{2}\right)} \\
& =\frac{H^{*}\left(\omega_{1}, \omega_{2}\right)}{\left|H\left(\omega_{1}, \omega_{2}\right)\right|^{2}+\alpha R\left(\omega_{1}, \omega_{2}\right)} .
\end{aligned}
$$

\section{APPENDIX E}

Proof of Proposition 2: Let $\boldsymbol{F}_{n}$ be the image matrix with elements $F_{i, j} ; i, j=1, \cdots, N$. By definition, $f_{n}$ is obtained by lexicographically ordering the elements of $\boldsymbol{F}_{n}$. Let $f_{i} ; i=1, \cdots, N^{2}$ be the elements of $f_{n}$. Then

$$
f_{N(i-1)+j}=F_{i, j} \forall 1 \leq i, j \leq N .
$$

By definition, $f_{s}$ is formed by ordering the columns of $\boldsymbol{F}_{n}$. Let $\boldsymbol{F}_{n}^{\prime}$ be the transpose of the image matrix with elements $F_{i, j}^{\prime} ; i, j=1, \cdots, N$. Obviously, $f_{s}$ can also be formed by lexicographically ordering the elements of $\boldsymbol{F}_{n}^{\prime}$. Let $f_{i}^{\prime} ; i=1,2, \cdots, N^{2}$ be the elements of $f_{s}$. Then

$$
f_{N(i-1)+j}^{\prime}=F_{i, j}^{\prime}=F_{j, i}=f_{N(j-1)+i} \forall 1 \leq i, j \leq N .
$$

The column vectors of $\boldsymbol{A}_{n}$ and $\boldsymbol{A}_{s}$ are related by (6.22), that is

$$
\boldsymbol{a}_{N(i-1)+j}^{\prime}=\boldsymbol{a}_{N(j-1)+i} \forall 1 \leq i, j \leq N .
$$

If $a_{i, j}^{\prime}$ and $a_{i, j} ; i, j=1, \cdots, N^{2}$ are the elements of $\boldsymbol{A}_{s}$ and $\boldsymbol{A}_{n}$, respectively, then

$$
\begin{gathered}
a_{l, N(i-1)+j}^{\prime}=a_{l, N(j-1)+i} \forall 1 \leq i, j \leq N ; \\
1 \leq l \leq N^{2} .
\end{gathered}
$$

Define $\boldsymbol{g}^{\prime}=\boldsymbol{A}_{s} \boldsymbol{f}_{s}$ and $\boldsymbol{g}=\boldsymbol{A}_{n} f_{n}$. Let $g_{i}^{\prime}$ and $g_{i} ; i=1$, $\cdots, N^{2}$ be the elements of $\boldsymbol{g}^{\prime}$ and $\boldsymbol{g}$, respectively. Taking into account (E2) and (E4)

$$
\begin{aligned}
g_{i}^{\prime} & =\sum_{k=1}^{N^{2}} a_{l, k}^{\prime} f_{k}^{\prime}=\sum_{i=1}^{N} \sum_{j=1}^{N} a_{l, N(i-1)+j}^{\prime} f_{N(i-1)+j}^{\prime} \\
& =\sum_{j=1}^{N} \sum_{i=1}^{N} a_{l, N(j-1)+i} f_{N(j-1)+i}=g_{l} \forall 1 \leq l \leq N^{2} .
\end{aligned}
$$

Therefore

$$
\boldsymbol{A}_{s} \boldsymbol{f}_{s}=\boldsymbol{g}^{\prime}=\boldsymbol{g}=\boldsymbol{A}_{n} \boldsymbol{f}_{n}
$$

which proves the proposition. 
Proof of Proposition 3: Assume that $\boldsymbol{A}_{n}$ is a separable matrix, that is, it can be written as follows:

$$
\boldsymbol{A}_{n}=\boldsymbol{C} \otimes \boldsymbol{D} .
$$

Let $\boldsymbol{a}_{i} ; i=1,2, \cdots, N^{2}$ be the columns of $\boldsymbol{A}_{n}$, while $\boldsymbol{c}_{j}$ and $\boldsymbol{d}_{j} ; j=1,2, \cdots, N$ are the columns of $\boldsymbol{C}$ and $\boldsymbol{D}$, respectively. Then

$$
\begin{aligned}
\boldsymbol{A}_{\boldsymbol{n}}= & {\left[\begin{array}{lllll}
\boldsymbol{a}_{1} \cdots & \boldsymbol{a}_{N} & \boldsymbol{a}_{N+1} & \cdots & \boldsymbol{a}_{2 N} \cdots \\
& \boldsymbol{a}_{N(N-1)+1} \cdots & \boldsymbol{a}_{N 2}
\end{array}\right] } \\
= & {\left[\begin{array}{llll}
\boldsymbol{c}_{1} \otimes \boldsymbol{d}_{1} \cdots & \cdots & \boldsymbol{c}_{1} \otimes \boldsymbol{d}_{N} & \boldsymbol{c}_{2} \otimes \boldsymbol{d}_{1} \cdots \\
& \boldsymbol{c}_{2} \otimes \boldsymbol{d}_{N} \cdots & \boldsymbol{c}_{N} \otimes \boldsymbol{d}_{1} \cdots \boldsymbol{c}_{N} \otimes \boldsymbol{d}_{N}
\end{array}\right] }
\end{aligned}
$$

where, in general

$$
a_{N(i-1)+j}=c_{i} \otimes d_{j} \forall 1 \leq i, j \leq N .
$$

The matrix $\boldsymbol{A}_{s}$, defined in Proposition 2 by $(6.21)$, is given by

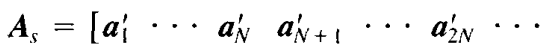

$$
\begin{aligned}
& \left.\boldsymbol{a}_{N(N-1)+1}^{\prime} \cdots \boldsymbol{a}_{N^{2}}^{\prime}\right] \text {. }
\end{aligned}
$$

Since, from Proposition 2, $\boldsymbol{a}_{N(i-1)+j}^{\prime}=\boldsymbol{a}_{N(j-1)+i}$

$$
a_{N(i-1)+j}^{\prime}=c_{j} \otimes d_{i} \forall 1 \leq i, j \leq N .
$$

Combining (E9) and (E10)

$$
\begin{aligned}
\boldsymbol{A}_{s}= & {\left[c_{1} \otimes \boldsymbol{d}_{1} \cdots \boldsymbol{c}_{N} \otimes \boldsymbol{d}_{1} \boldsymbol{c}_{1} \otimes \boldsymbol{d}_{2} \cdots\right.} \\
& \left.\boldsymbol{c}_{N} \otimes \boldsymbol{d}_{2} \cdots \boldsymbol{c}_{1} \otimes \boldsymbol{d}_{N} \cdots \boldsymbol{c}_{N} \otimes \boldsymbol{d}_{N}\right] \\
= & {\left[\boldsymbol{C} \otimes \boldsymbol{d}_{1} \quad \boldsymbol{C} \otimes \boldsymbol{d}_{2} \cdots \boldsymbol{C} \otimes \boldsymbol{d}_{N}\right] . }
\end{aligned}
$$

Let $c_{i}^{T} ; i=1, \cdots, N$ be the rows of $C$. From (E11)

$$
\begin{aligned}
& \boldsymbol{A}_{s}=\left[\begin{array}{cccc}
c_{1}^{T} \otimes d_{1} & c_{1}^{T} \otimes d_{2} & \cdots & c_{1}^{T} \otimes d_{N} \\
c_{2}^{T} \otimes d_{1} & c_{2}^{T} \otimes d_{2} & \cdots & c_{2}^{T} \otimes d_{N} \\
\vdots & \vdots & \vdots & \vdots \\
c_{N}^{T} \otimes d_{1} & c_{N}^{T} \otimes d_{2} & \cdots & c_{N}^{T} \otimes d_{N}
\end{array}\right]
\end{aligned}
$$

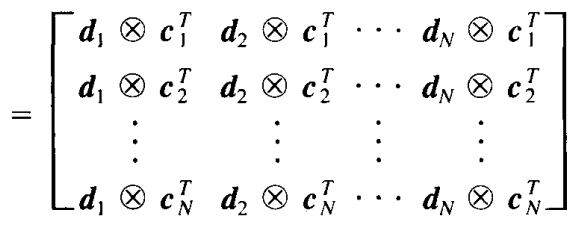

$$
\begin{aligned}
& =\left[\begin{array}{c}
{\left[d_{1} \cdots d_{N}\right] \otimes c_{1}^{T}} \\
{\left[d_{1} \cdots d_{N}\right] \otimes c_{2}^{T}} \\
\vdots \\
{\left[d_{1} \cdots d_{N}\right] \otimes c_{N}^{T}}
\end{array}\right]=\left[\begin{array}{c}
D \otimes c_{1}^{T} \\
D \otimes c_{2}^{T} \\
\vdots \\
D \otimes c_{N}^{T}
\end{array}\right]
\end{aligned}
$$

which proves the proposition.
Proof of Proposition 3: Let $\boldsymbol{c}_{i}^{T}, \boldsymbol{d}_{i}^{T} ; \boldsymbol{i}=1, \cdots, N$ be the rows of $C$ and $D$, respectively. From (E12)

$$
\begin{aligned}
& A_{s}^{T}=\left[\begin{array}{llll}
D^{T} \otimes c_{1} & D^{T} \otimes c_{2} & \cdots & D^{T} \otimes c_{N}
\end{array}\right] \\
& =\left[d_{1} \otimes c_{1} \cdots d_{N} \otimes c_{1} \quad d_{1} \otimes c_{2} \cdots\right. \\
& \left.\boldsymbol{d}_{N} \otimes c_{2} \cdots \boldsymbol{d}_{1} \otimes c_{N} \cdots \boldsymbol{d}_{N} \otimes c_{N}\right] \\
& =\left[\begin{array}{llllll}
\boldsymbol{b}_{1} & \cdots & \boldsymbol{b}_{N} & \boldsymbol{b}_{N+1} & \cdots & \boldsymbol{b}_{2 N}
\end{array} \cdots\right. \\
& \left.\boldsymbol{b}_{N(N-1)+1} \cdots \boldsymbol{b}_{N^{2}}\right]
\end{aligned}
$$

where

$$
b_{N(i-1)+j}=d_{j} \otimes c_{i} \forall 1 \leq i, j \leq N .
$$

Let $g^{\prime}$ be the vector consisting of the ordered columns of G. According to Proposition 2

$$
\boldsymbol{g}_{R}=A_{s}^{T} \boldsymbol{g}=\boldsymbol{A}_{s}^{\prime T} \boldsymbol{g}^{\prime}
$$

where

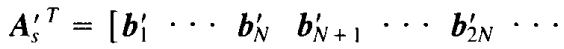

$$
\begin{aligned}
& \left.\boldsymbol{b}_{N(N-1)+1}^{\prime} \cdots \boldsymbol{b}_{N^{2}}^{\prime}\right]
\end{aligned}
$$

and $\boldsymbol{b}_{i}^{\prime} ; i=1, \cdots, N^{2}$ are related to $\boldsymbol{b}_{i} ; i=1, \cdots$, $N^{2}$ by $\boldsymbol{b}_{N(i-1)+j}^{\prime}=\boldsymbol{b}_{N(j-1)+i}$, or equivalently

$$
\boldsymbol{b}_{N(i-1)+j}^{\prime}=\boldsymbol{d}_{i} \otimes c_{j} \forall 1 \leq i, j \leq N .
$$

Combining (E16) and (E17)

$$
\begin{aligned}
& \boldsymbol{A}_{s}^{\prime r}=\left[d_{1} \otimes c_{1} \cdots d_{1} \otimes c_{N} \quad d_{2} \otimes c_{1} \cdots\right. \\
& \left.\boldsymbol{d}_{2} \otimes \boldsymbol{c}_{N} \cdots \boldsymbol{d}_{N} \otimes \boldsymbol{c}_{1} \cdots \boldsymbol{d}_{N} \otimes \boldsymbol{c}_{N}\right]
\end{aligned}
$$

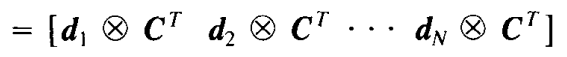

$$
\begin{aligned}
& =D^{T} \otimes C^{T} \text {. }
\end{aligned}
$$

Hence

$$
\boldsymbol{g}_{R}=\boldsymbol{D}^{T} \otimes \boldsymbol{C}^{T} \boldsymbol{g}^{\prime} .
$$

Since $g^{\prime}$ can also be obtained by lexicographically ordering the elements of $G^{T}$, (E19) can be written in a matrix form as follows:

$$
\boldsymbol{G}_{R}=\boldsymbol{D}^{T} \boldsymbol{G}^{T} \boldsymbol{C}
$$

\section{REFERENCES}

[1] H. C. Andrews and B. R. Hunt, Digital Image Restoration. Englewood Cliffs, NJ: Prentice-Hall, 1977.

[2] W. K. Pratt, Digital Image Processing. New York: Wiley, 1978.

[3] A. N. Tikhonov and V. Y. Arsenin, Solutions of Ill-Posed Problems. New York: Wiley, 1977.

[4] B. R. Hunt, "Image restoration," in Digital Image Processing Techniques, M. P. Ekstrom, Ed. New York: Academic, 1984, pp. 5376.

[5] M. Z. Nashed, "Aspects of generalized inverses in analysis and regularization," in Generalized Inverses and Applications, M. Z. Nashed. Ed. New York: Academic, 1976.

[6] N. B. Karayiannis and A. N. Venetsanopoulos, "Regularization theory in image restoration: The regularizing operator approach," Opt. Eng. . vol. 28, no. 7, pp. 761-780, July 1989. 
[7] H. S. Hou and H. C. Andrews, "Cubic splines for image interpolation and digital filtering," IEEE Trans. Acoust., Speech, Signal Proressing. vol. ASSP-26, pp. 508-517, Dec. 1978.

[8] A. K. Katsaggelos, J. Biemont, R. M. Mersereau, and R. W. Shafer, *A generalized formulation of constrained iterative restoration algorithms, " in Proc. ICASSP'85. Tampa, FL, 1985, pp. 700-703.

[9] B. R. Hunt, "The application of constrained least squares estimation to image restoration by digital computer," IEEE Trans. Comput., vol. C-23, pp. 805-812, Sept. 1973.

[10] B. R. Frieden, "Restoring with maximum likelihood and maximum entropy," J. Opt. Soc. Amer., vol. 62, pp. 511-518, 1972.

[11] S. J. Wernecke and L. R. D'Addario, "Maximum entropy image reconstruction," IEEE Trans. Comput., vol. C-26, pp. 351-364, Apr. 1977.

[12] F. Glazer, "Multilevel relaxation in low-level computer vision." in Multilevel Image Analysis, A. Rosenfeld, Ed. New York: SpringerVerlag.

113] V. Torre and T Poggio, "On edge detection," IEEE Trans. Pattern Anal. Machine Inteil., vol. PAMI-8, pp. 147-163, Mar. 1986.

[14] J. Canny, "A computational approach to edge detection," IEEE Trans. Pattern Anal. Machine Intell., vol. PAMI-8, pp. 679-698, Nov. 1986.

[15] A. K. Katsaggelos and B. J. Sullivan, "Regularized edge detection," in Proc. ICASSP '88, New York, 1988, pp. 1048-1051.

[16] B. K. P. Horn and B. G. Schunk, "Determining optical flow," Artificial Intell., vol. 17, pp. 185-203, 1981.

[17] B. K. P. Horn, "Obtaining shape from shading information," in The Psychology of Computer Vision. P. H. Winston, Ed. New York: McGraw-Hill, 1975, pp. 115-155.

18] W. E. L. Grimson. "A computational theory of visual surface interpolation," Artificial Intell. Lab. Memo 613, M.I.T., 1981.

[19] —- From Images to Surfaces: A Computational Study of the Human Early Visual System. Cambridge, MA. M.I.T. Press, 1981.

[20] — - "An implementation of a computational theory of visual surface interpolation," Comput. Vision, Graphics, Image Processing, vol. 22 , pp. 39-69, 1983

[21] M. Brady and B. K. P. Horn, "Rotational symmetric operators for surface interpolation," Comput. Vision, Graphics, Image Processing. vol. 22 , pp. 70-94, 1983.

[22] D. Terzopoulos, "Multilevel reconstruction of visual surfaces: Variational principles and finite element representations." Artificial Intell. Lab. Memo 671, M.I.T., 1982.; reprinted in Multiresolution Image Processing and Analysis, A. Rosenfeld, Ed. New York: Springer-Verlag, 1984, pp. 273-310.

[23] _ " "Multiresolution algorithms in computational vision," in Image Understanding, S. Ullman and W. Richard, Eds. Albex, 1984, pp. $225-262$.

[24] - . "Regularization of inverse visual problems involving discontinuities," IEEE Trans. Pattern Anal. Machine Intell., vol. PAMI-8, pp. 413-424. July 1986.

[25] T. Pavlidis. "A critical survey of image analysis methods," in Proc. Int. Conf. Artificial Intell. . Paris, 1986, pp. 502-511.

[26] A. N. Venetsanopoulos, "Digital image processing and analysis," in Signal Processing. T. S. Durrani and J. L. Stora. Eds. Amsterdam. The Netherlands: North-Holland, 1987.

[27] I. M. Gelfand and S. V. Fomin, Calculus of Variations. Englewood Cliffs, NJ: Prentice-Hall, 1963

[28] H. Sagan. Introduction to the Calculus of Variations. New York: McGraw-Hill, 1969.

$29]$ N. B. Karayiannis, "Regularization theory in image restoration," M.A.Sc. thesis, Univ. Toronto. Jan. 1987.

[30] H. L. Weinert and T. Kailath. "Stochastic interpretation and recursive algorithms for spline functions," Ann. Statistics, vol. 2, no. 4. pp. 787-794, 1974

[31] G. S. Kimeldorf and G. Wahba, "A correspondence between Bayesian estimation and smoothing by splines." Ann. Math. Statistics, vol. 41 , no. 2, pp. 495-502, 1970.

[32] T. C. Chen and R. J. P. DeFiguiredo "Two-dimensional interpolation by generalized spline filters based on partial differential equation image models," IEEE Trans. Acoust., Speech. Signal Processing. vol. ASSP-33, pp. 631-642, June 1985

133] N. B. Karayiannis and A. N. Venetsanopoulos. "Comments on twodimensional interpolation by generalized spline filters based on partial differential equation image models," IEEE Trans. Acoust., Speech, Signal Processing, vol. ASSP-35, pp. 1778-1779, Dec. 1987.
[34] - , "Image interpolation based on variational principles," Signal Processing, under review.

[35] L. L. Shumaker, "Fitting surfaces to scattered data," in Approximation II, G. G. Lorentz, C. K. Chui, and L. L. Shumaker, Eds New York: Academic, 1976, pp. 203-267.

[36] R. Franke, "Scattered data interpolation: Tests of some methods," Math. Comput., vol. 38, pp. 181-199, 1982.

[37] I. S. Sokolnikoff, Mathematical Theory of Elasticity. New York: McGraw-Hill, 1956.

[38] N. B. Karayiannis and A. N. Venetsanopoulos, "Image restoration: A regularization approach," in Proc. Int. Conf. Image Processing Appl., London, England, June 24-26, 1986, pp. 1-5.

[39] 1. Gladwell and R. Wait, Eds., A Survey of Numerical Methods for Partial Differential Equations. Oxford, U.K.: Clarendon, 1979.

[40] O. C. Zienkiewicz, The Finite Element Method, 3rd Ed. New York: McGraw-Hill, 1977.

[41] R. W. Schafer, R. M. Mersereau, and M. A. Richards, "Constrained iterative restoration algorithms," Proc. IEEE, vol. 69, pp. 432-450, Apr. 1981.

[42] K. Miller, "Least squares methods for ill-posed problems with a prescribed bound," SIAM J. Math. Anal., vol. I, no. 1, pp. 52-74, Feb. 1970 .

[43] N. B. Karayiannis and A. N. Venetsanopoulos, "Regularization theory, MAP estimation and Wiener filtering in image restoration: Points of tangency and areas of intersection," in Proc. $1987 \mathrm{Int}$. Symp. Circuits Syst., Philadelphia, PA, May 4-7, 1987, pp. 835-838.

[44] L. H. Van Trees, Detection, Estimation and Modulation Theory, Part I. New York: Wiley, 1968

[45] A. K. Jain, "Partial differential equations and finite-difference methods in image processing, Part 1: Image representation." J. Optimiz. Theory Appl., vol. 23, no. 1, pp. 65-91, Sept. 1977.

[46] N. B. Karayiannis and A. N. Venetsanopoulos, "A class of generalized regularizing filters for image restoration," in Proc MONTECH'87, Montreal, P.Q. Canada, Nov. 1987.

[47] D. G. Luenberger, Introduction to Linear and Nonlinear Program ming. Reading, MA: Addison-Wesley, 1973.

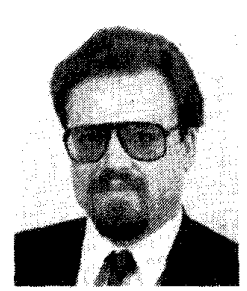

Nicolaos B. Karayiannis ( $\left.S^{\prime} 85\right)$ was born in Greece on January 1, 1960. He received the Diploma degree in electrical engineering from the National Technical University of Athens in 1983. and the M.A.Sc. degree in electrical engineering from the University of Toronto, Toronto, Ont., Canada, in 1987.

From 1983 to 1984 he was a Research Assistant in the Nuclear Research Center "Democritos," Athens, Greece, where he was engaged in research on multidimensional signal processing. He is currently working toward the Ph.D. degree at the University of Toronto, where he is also a Research and Teaching Assistant. His current research interests include image processing, image analysis, and neural networks.

Mr. Karayiannis is a member of the International Neural Network Society (INNS), the International Society for Optical Engineering, and the Technical Chamber of Greece.

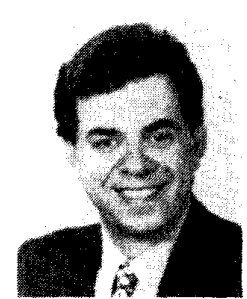

Anastasios N. Venetsanopoulos (S'66-M'69$S^{\prime} M^{\prime} 79-F^{\prime} 88$ ) received the $B . S$. degree from the National Technical University of Athens (NTU). Greece, in 1965, and the M.S., M.Phil., and $\mathrm{Ph} . \mathrm{D}$. degrees in electrical engineering, all from Yale University, in 1966, 1968, and 1969, respectively.

$\mathrm{He}$ joined the University of Toronto. Canada, in September 1968, where he is now Professor in the Department of Electrical Engineering. He also served as Chairman of the Communications Group (1974 to 1978 and 1981 to 1986), and as Associate Chairman of the Department of Electrical Engineering (1978 to 1979). He was on research leave at the Swiss Federal Institute of Technology, the University of Flor- 
ence, the Federal University of Rio de Janeiro, the National Technical University of Athens, and the Imperial College of Science and Technology, and was Adjunct Professor at Concordia University. He served as Lecturer of numerous short courses to industry and continuing education programs: he is a contributor to eleven books and has published over 300 papers in digital signal and image processing, and digital communications; he also served as consultant to several organizations, and as Editor of the Canadian Electrical Engineering Journal (1981 to 1983).

Dr. Venetsanopoulos has served the IEEE in the following capacities. IEEE Activities-Boards: IEEE Educational Activities Board (1987). Re gions: Region 7, Educational Activities Committee Chairman (1986), Nominations and Appointments Committee (1982). Councils: Central Canada Council: Chairman (1981-1982), Past Chairman and Nominations Committee Chairman (1983-1984). Sections: Toronto; Chairman (19771979), Vice Chairman (1976-1977), Educational Activities Coordinator (1974-1976), Past Chairman and Nominations Committee Chairman (19791981). Societies: Circuits and Systems: Associate Editor in Digital Signal Processing, IEEE TRANSACTIONS ON CiRCuIrs AND Systems (June 1985-
May 1987); Guest Editor, Special Issue on Digital Image Processing and Applications, IEEE Transactions on ClRcuits and Systems (November 1987). Conferences: Canadian Communications and Energy Conference, Program Committee $(1978,1982)$. Electronicon, Program Committee $(1971,1973,1975,1983,1985)$. International Conference on Communications, Program Chairman (1978, 1986). International Symposium on Circuits and Systems, Finance Chairman (1983). International Conference on Acoustics, Speech, and Signal Processing, Program Chairman (1991). Representative: IEEE/CSEE Joint Committee, Region 7 (1983-1984). He was President of the Canadian Society for Electrical Engineering and VicePresident of the Engineering Institute of Canada (1983-1984). He was a Fulbright Scholar, A. F. Schmitt Scholar, and recipient of the J. Vakis Award. He is a member of the New York Academy of Sciences, Sigma Xi, the International Society for Optical Engineering, and the Technical Chamber of Greece; he is a Registered Professional Engineer in Ontario and Greece, a Fellow of the Engineering Institute of Canada, and a Fellow of the Institute of Electrical and Electronics Engineers for contributions to digital signal and image processing. 